AD/RHIC-PH-24

Detection of Jets with Calorimeters

at Hadron Colliders

T. Akesson, M. Albrow, P.N. Burrows, T. Cox, J.P. de Brion, C. Fabjan, M. Holder, G. Ingelman, G. Jarlskog, K. Meier, P.G. Rancoita, J. Russ, J. Schukraft, G. Stevenson, G: Stevenson, A. Weidberg and $R$. Wigmans

June 16, 1987 


\title{
DETECTION OF JETS WITH CALORIMETERS AT HADRON COLLIDERS
}

T. Ȧkesson, M. Albrow, P.N. Burrows, T. Cox, J.P. de Brion, C. Fabjan, M. Holder, G. Ingelman, G. Jarlskog, K. Meier, P.G. Rancoita, J. Russ, J. Schukraft, G. Stevenson, A. Weidberg and R. Wigmans

\begin{abstract}
The results presented here are from the Jet-Calorimeter Working Group set up by the Physics and Detector Advisory Panel, in the framework of the CERN Long Range Planning Committee. A small-radius silicon calorimeter is suggested for a high-luminosity pp machine. The detector performance is demonstrated and the radiation damage is . calculated. Calorimetrization of the fragmentation region is discussed.
\end{abstract}

\section{INTRODUCTION}

The Large Hadron Collider, LHC, and the Superconducting Super Collider, SSC, have been studied on several occasions [1, 2], and many important questions regarding both a non-defined calorimeter [1] and one having a rather conventional design [2] have been addressed and solved by the working groups concerned. In this study, we have investigated a new, technologically advanced detector.

This paper contains the following sections:

2. Jet characteristics. The properties of jets at these energies are discussed.

3. The calorimeter Monte Carlo. The Monte Carlo used for examining the detector performance is described. 
4. The small-radius Si calorimeter. A new detector design for the LHC (SSC) is suggested and discussed.

5. Dose to the U/Si calorimeter. A calculation of the estimated radiation hazard at the LHC is presented.

6. Performance of the small-radius calorimeter. The performance of the suggested detector is demonstrated for a test-case.

7. Forward calorimeter and total energy measurement. The total energy measurement and calorimetry in the high-radiation level of the forward region are discussed.

\section{Conclusions.}

\section{JET CHARACTERISTICS}

Many of the phenomena that will be of primary interest at future accelerators will be observable only in terms of final-state jets, e.g. new massive states are often expected to decay into high-energy quarks and gluons giving rise to jets of particles. One would therefore like to measure the four-vectors of jets and, for example, to search for such new states through resonances in the invariant masses of combinations of jets. In order to assess how well this can be done using calorimetric techniques, it is important to have a reasonable estimate of the basic jet properties, since the calorimeter response will depend, for instance, on whether the jet energy is carried by a few hard particles or many soft particles. The angular width of a jet is also of interest in relation to the granularity of the calorimeter. We have thus made a detailed study of such jet properties, reported separately in Ref. [3], and summarize a few of the main results here.

Models for the perturbative evolution of the jet have evolved considerably in the last few years. The treatment in terms of a dynamical simulation, using the QCD parton cascade approach giving rise to multiple gluon emission, has been found to produce sizeable effects relative to the available exact matrix element calculations at low order. Combined with phenomenological models for the non-perturbative hadronization process, such as the string or cluster decay models, the perturbative cascade approach has been shown (see references in [3]) to be in good agreement with observations at the highest $\mathrm{e}^{+} \mathrm{e}^{-}$energies and at the CERN p $\overline{\mathrm{p}}$ Collider. This provides a good starting point for realistic extrapolations to future energies, where gluon radiation from highly virtual hard-scattered partons will be abundant. This is further supported by the generally good agreement between different such models, as well as by some comparisons with analytical QCD calculations.

In order to produce useful jet properties, the concept of a jet has to be defined in an experimentally realistic way; theoretical parton-level results are only of very limited value for our needs. We therefore base our results on a currently used jet algorithm in terms of the energy flow in an idealized, but realistic, calorimeter. The calorimeter granularity and the basic jet-width parameter do, in fact, influence the number of reconstructed jets as well as their detailed properties. However, at the TeV energy scale we find a small sensitivity to many other parameters, such as whether jets are reconstructed by summing cell energies or cell 'momentum vectors'.

The hardness of a jet can be illustrated by the property of $1 \mathrm{TeV}$ quark jets (at CLIC) to have $50 \%(10 \%)$ of their energy carried by particles having E $>80(300) \mathrm{GeV}$. The width of such jets is shown by $50 \%$ of the particles and $50 \%$ of the energy within $3^{\circ}$ and $2^{\circ}$, respectively, of the reconstructed jet axis. The charged-particle multiplicity is expected to be around 25 for such a jet and 78 in the whole event. In terms of longitudinal fragmentation, gluon jets are considerably softer than quark jets - but not necessarily much wider - at very large energies.

The measured mass of a jet depends both on the virtual mass of the parton emerging from the hard process, which then gives rise to the jet evolution, and on how much of the resulting jet cascade is included in the reconstructed jet, which depends on the size of the jet cone used in the algorithm. It is not possible to find heavy-quark jets, e.g. top, just from the reconstructed jet mass, since off-shell light quarks will in fact give rise to a similar jet-mass distribution. Because of this, and of the resulting gluon bremsstrahlung, the general properties of t-quark jets will also not differ much from light-quark jets at this energy scale. A distinctive top signature is provided by leptons from a semileptonic decay; however, this requires that a sufficiently large $p_{T}$ be demanded in order to eliminate leptons from decays of lighter flavours. 


\section{THE CALORIMETER MONTE CARLO}

A simple parametrization of the hadronic showers was used to simulate the calorimeter response. The simulation used an empirical parametrization [4] for the longitudinal shower development and a Gaussian distribution for the transverse energy spread. The width $\sigma$ of the transverse size of the hadronic shower has a linear growth for the first $1.5 \lambda$ and then stays constant. The $\sigma$ of the transverse size of the electromagnetic shower has a linear growth for the duration of the shower. The starting point of the shower was generated with an exponential distribution with the absorption length $\lambda$ for hadronic showers. The energy loss of charged particles between the beginning of the calorimeter and the starting-point of the shower was taken into account. The calorimeter response to this $\mathrm{dE} / \mathrm{dx}$ loss is amplified by a $\mu / \mathrm{e}$ ratio $=1.3$. The response to the energy deposition of the hadronic shower is suppressed by the measured asymptotic $\mathrm{e} / \mathrm{h}$ ratio $(1.0)$.

The geometry of the detector was coded using the GEANT3 package [5].

The parameters were tuned to agree with UA2 test beam data [6], with $\pi$ scans across the face of a calorimeter. The calorimeter for the test-beam set-up consists of three slices of the UA2 central calorimeter [6] corresponding to an array of ten cells in $\theta$ by three cells in $\phi$. The cell size at the front face of the calorimeter is $\approx 10 \mathrm{~cm} \times 15 \mathrm{~cm}$. The calorimeter is divided into three longitudinal sections of $0.5,2$, and 2 nuclear absorption lengths, respectively.

The energy flow in the $\theta$ direction was used to tune the transverse width in the shower parametrization. The results are shown in Fig. 1 for different beam impact points $\theta_{\text {beam. }}$. In order to check that the fluctuations in the transverse shower development were correctly simulated, the shower energy weighted centre was calculated: $\theta=\Sigma E_{i} \theta_{i} / \Sigma E_{i}$. The r.m.s. of $\theta$ was used as a measure of the fluctuations. The results for different values of $\theta_{\text {beam }}$ are given in Table 1. The fraction of energy deposited in the different longitudinal compartments was used to tune the longitudinal shower parametrization (energy-independent) to agree with $10 \mathrm{GeV} / \mathrm{c}$ pion data. However, this did

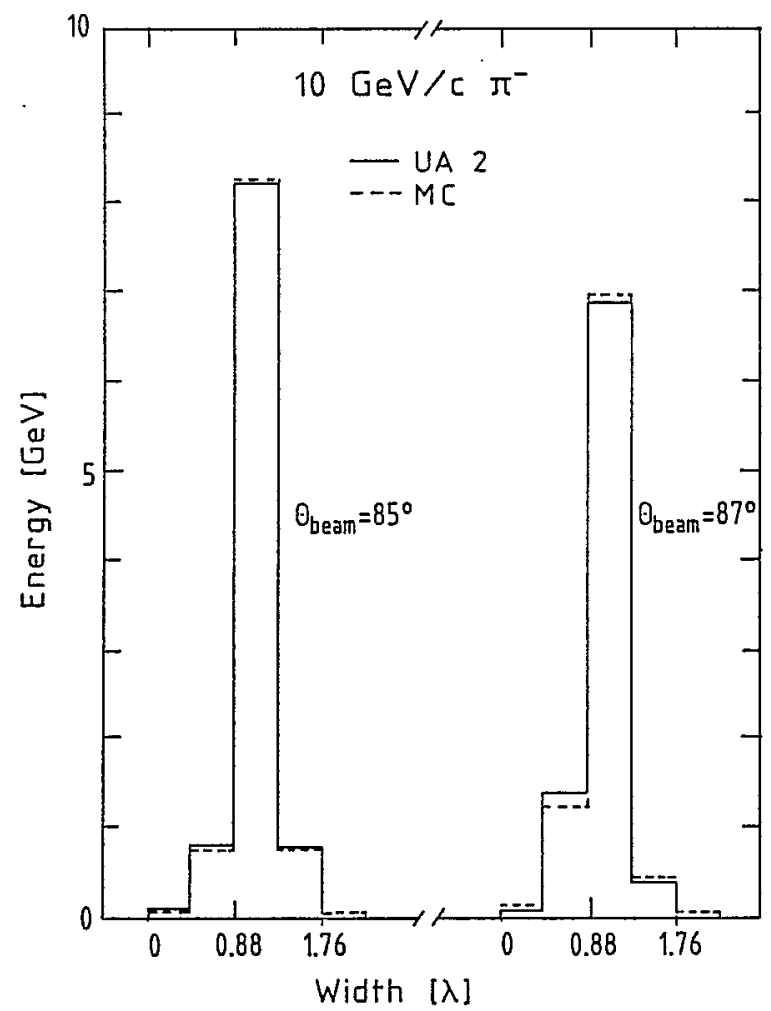

Table 1

The r.m.s. of energy-weighted centres

\begin{tabular}{|c|c|c|}
\hline \multirow{2}{*}{$\begin{array}{c}\theta_{\text {beam }} \\
\left({ }^{\circ}\right)\end{array}$} & \multicolumn{2}{|c|}{ r.m.s. of $\Theta$} \\
\cline { 2 - 3 } & Data & Monte Carlo \\
\hline 85 & 1.33 & 1.31 \\
87 & 1.43 & 1.47 \\
89 & 1.77 & 1.65 \\
\hline
\end{tabular}

Fig. 1 Comparison of the lateral hadronic showersize given by the UA2 test-beam data [6] with that of the simulation program 
not give a good fit to higher-energy data, and more work would be needed to produce an energy-dependent parametrization. In conclusion, the Monte Carlo should give a reasonable simulation of the transverse shower spread, but it contains only a very crude treatment of the longitudinal shower development. For the test case addressed in Section 6, a realistic simulation of the transverse shower spread is, of course, more essential.

\section{THE SMALL-RADIUS Si CALORIMETER}

\subsection{Introduction}

Previous hadron collider studies (Lausanne and SSC Workshops) [1, 2] have examined hadron calorimeters based on scintillator or liquid-argon readout. The UA1 upgrading program is pushing warm-liquid readout from the status of idea to an actual demonstration of technical feasibility [7]. This study has chosen to use silicon as the readout medium in a uranium-plate sampling calorimeter, based on the potential of this material for stable electronics-limited gain calibration, simple mechanical assembly with few dead zones, and an almost unlimited segmentation potential. The pros and cons for silicon are listed below:

Advantages

Absolute gain for the sampling medium

Gain adjustment and monitoring

by radioactive sources

Non-saturating readout, so sources give absolute energy scale

Fast charge collection

Fine lateral and longitudinal segmentation, easy
Disadvantages

Small sampling fraction

Expensive sampling material

Susceptible to radiation damage

and feasible

\subsection{Calorimeter performance requirements}

For hadron calorimetry at the LHC or at other ultra-high-energy colliders, the exact matching of calorimeter response for electromagnetic and hadronic shower components is crucial if we are to avoid a non-linear variation of energy resolution with the number of sampling charges. The constant term in the energy resolution function (among other effects) arises from departures from the ratio $\mathrm{e} / \mathrm{h}=1.0$, where $\mathrm{e} / \mathrm{h}$ is the ratio of the response to electrons and hadrons of the same energy that is incident on the calorimeter [8]. Any non-zero constant term will limit the energy resolution to a few percent at energies of several hundred $\mathrm{GeV}$, rather than give the expected ' $\sqrt{\mathrm{E}}$ ' improvement. A recent study [8] (exploiting the original ideas of Ref. [9]) of the role of fission neutrons in compensating for fluctuations in nuclear binding-energy losses during hadron cascades, has demonstrated the fundamental need to detect low-energy fission and/or spallation neutrons in order to achieve the 'compensation' condition, $\mathrm{e} / \mathrm{h}=1.0$. This requires the presence of an optimal density of protons in the system to transform neutron kinetic energy into a signal which can be detected in the sampling medium. In order to achieve this for low-energy fission neutrons, the protons must be in intimate contact with the sampling medium because of the short proton range. In scintillator this occurs automatically. For liquid-argon readout, there is no compensation because there is no hydrogen - it is probable that not enough methane can be added as a dopant to achieve this compensation [10].

For silicon sampling the question is how to introduce hydrogenous material. Bare U/Si calorimeters would have $\mathrm{e} / \mathrm{h}>1.1$ according to Ref. [8]. However, the range of recoil protons is long enough so that covering the silicon detectors with a thin polyethylene film $(100 \mu \mathrm{m})$ should give enough neutron conversions to produce compensation. A fact that is crucial to this assumption is that silicon responds linearly to the slow protons produced, whilst most other media saturate and see only about $20 \%$ of the actual recoil proton energy. An initial examination of this possibility for the model described in Ref. [8] indicated that indeed one can achieve $\mathrm{e} / \mathrm{h}=1.0 \mathrm{in}$ a uranium/silicon calorimeter by this means. 
In order to evaluate the calorimeter physics potential, a simulation has been made for the process $\mathrm{W} \rightarrow$ jet + jet. The calorimeter simulation program used for this purpose is described in Section 3 , and the result of the simulation is given in Section 6.

\subsection{Silicon calorimetry}

There is already a great deal of information available on silicon readout of electromagnetic sampling calorimeters. In this case, the neutron response is irrelevant. The arguments given for using silicon rather than other sampling media are

i) high effective density, leading to good shower confinement;

ii) good long-term gain stability, calibrated absolutely by radioactive sources - in nuclear physics applications, $\gamma$-spectroscopy detectors routinely maintain a gain stability of better than $0.3 \%$, limited not by the silicon itself but by electronic gain stability and calibration;

iii) simple mechanical assembly considerations.

The data from these tests strongly support the view that silicon detectors are an excellent choice for stable calorimetry. Very little $\left(<1 \%\right.$ ) change was observed over months of operation of a $24 \mathrm{X}_{0} \mathrm{~W} / \mathrm{Si}$ electromagnetic calorimeter using undepleted, low-resistivity silicon detectors. The energy resolution was found to be $\sigma(\mathrm{E}) / \mathrm{E}=$ 17.6 $\sqrt{ }(\tau / E)$, where $\tau$ is the sampling frequency [11]. Based on these studies, the SICAPO Collaboration is constructing a prototype of a U/Si hadronic calorimeter with which to study the conditions required for a compensating silicon hadron calorimeter [12]. The active silicon area of the calorimeter will be $6.5 \mathrm{~m}^{2}$.

Our working group has concentrated on several basic questions regarding the design and performance of a $\mathrm{Si}$ sampling calorimeter:

i) What is the minimum inner radius for the calorimetry, compatible with the aim of reconstructing high-energy massive states decaying into quark jets?

ii) What is the minimal segmentation, lateral and longitudinal, which will give good jet reconstruction potential with minimal overlap confusion?

iii) What electromagnetic segmentation can be achieved?

iv) Can one make a hermetically sealed calorimeter?

v) What is the radiation environment? Can silicon survive?

vi) What would be the cost of a Si calorimeter?

\subsection{COSICA - The COmpact Sllicon CAlorimeter}

The idea behind the small inner radius is that a calorimetric jet detector does not need an inner tracking chamber in order to do TeV-mass-scale jet physics. If the major job of the detector is to measure jet energies and angles in an optimal fashion and to provide strong lepton identification, then it seemed to us that a compact, high-density calorimeter starting close to the beam would have numerous advantages. We expect that it will be absolutely necessary to incorporate a microvertex detector to isolate the interaction point, and we suggest that a compact transition radiation detector (TRD) [13] should follow immediately to give a quick electron tag for triggers. It is assumed that these two devices will occupy the first $20 \mathrm{~cm}$ of the radius from the beam line. Thus the electromagnetic section of COSICA begins at a $20 \mathrm{~cm}$ inner radius. The basic design is a cylinder with end caps, since hermeticity is needed for all $\theta>10^{\circ}$, according to the Working Groups on Standard Theory Physics and on New Physics. This simple design idea is modified slightly in the actual calorimeter geometry shown in Fig. 2.

This calorimeter layout has the barrel region covering angles down to $30^{\circ}$. The effective variation in plate thickness is then a factor of 2 , going from $90^{\circ}$ to $30^{\circ}$. This worsens the expected energy resolution (because of sampling fluctuations) from $0.46 / \sqrt{\mathrm{E}}$ at $90^{\circ}$ to $0.53 / \sqrt{\mathrm{E}}$ at $30^{\circ}$ according to Ref. [8], but since the energy will increase, this seems to be acceptable. The two additional barrel segments at smaller angles are chosen so that the 


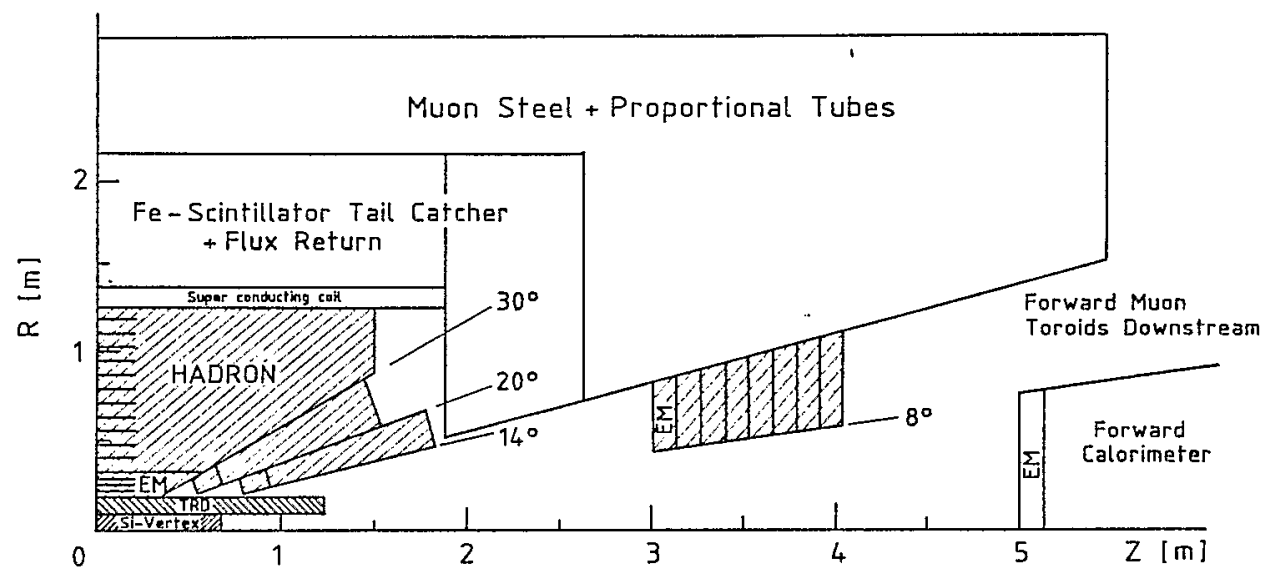

Fig. 2 COSICA - The COmpact SIlicon CAlorimeter. The figure shows a complete jet calorimeter apparatus for the LHC: COSICA (shaded area) plus a central vertex detector and TRD, with external magnet coil and muon steel for $4 \pi$ coverage.

maximum variation of effective plate thickness is less than a factor of 2 . The limit to the angular coverage using the high-quality $\mathrm{U} / \mathrm{Si}$ calorimetry is at $7^{\circ}$, owing to the radiation level near the beam pipe; this will be discussed later.

A calorimeter for an LHC detector must meet two fundamental conditions: the construction cost must be acceptable, and its operating lifetime in the LHC (SSC) radiation environment must be long compared with one operating year at full luminosity. The fulfilment of these requirements is discussed in later subsections.

\subsection{Electromagnelic section}

The proposed parameters are given in Table 2. The lateral segmentation in the electromagnetic section is $2 \mathrm{~cm}$ $\times 2 \mathrm{~cm}$ cells, and the total depth is $20 \mathrm{X}_{0}$. Because high-energy electromagnetic showers are much more sharply

Table 2

Parameters for the Si calorimeter

\begin{tabular}{|l|l|l|}
\cline { 2 - 3 } \multicolumn{1}{c|}{} & Electromagnetic section & \multicolumn{1}{c|}{ Hadronic section } \\
\hline Absorber & $2 \mathrm{~mm} \mathrm{U}$ & $4 \mathrm{~mm} \mathrm{U}$ \\
Readout & $0.4 \mathrm{~mm} \mathrm{Si}+0.1 \mathrm{~mm} \mathrm{CH}_{2}$ & $0.4 \mathrm{~mm} \mathrm{Si}+0.1 \mathrm{~mm} \mathrm{CH} 2$ \\
Cell thickness & $2.5 \mathrm{~mm}$ & $4.5 \mathrm{~mm}$ \\
Longitudinal segment & 8 cells $/ 5 \mathrm{X}_{0}$ & 13 cells $/ 0.5 \lambda$ \\
Number of segments & 4 & 12 \\
Transverse cell size & $2 \mathrm{~cm} \times 2 \mathrm{~cm}$ & $2 \mathrm{~cm} \times 2 \mathrm{~cm}$ \\
Silicon strip & & \\
Position sample: & & \\
depth & $(5,10$, and 20$) \mathrm{X}_{0}$ & $(0.5,1.0$, and 1.5$) \lambda$ \\
pitch & $0.5 \mathrm{~mm}$ & $0.5 \mathrm{~mm}$ \\
Energy resolution & $15 \% / \sqrt{\mathrm{E}}$ & $46 \% / \sqrt{\mathrm{E}}$ \\
e/h ratio & 1.0 & 1.0 \\
Spatial resolution & $0.2 \mathrm{~mm}$ & $2 \mathrm{~mm}$ \\
\hline
\end{tabular}




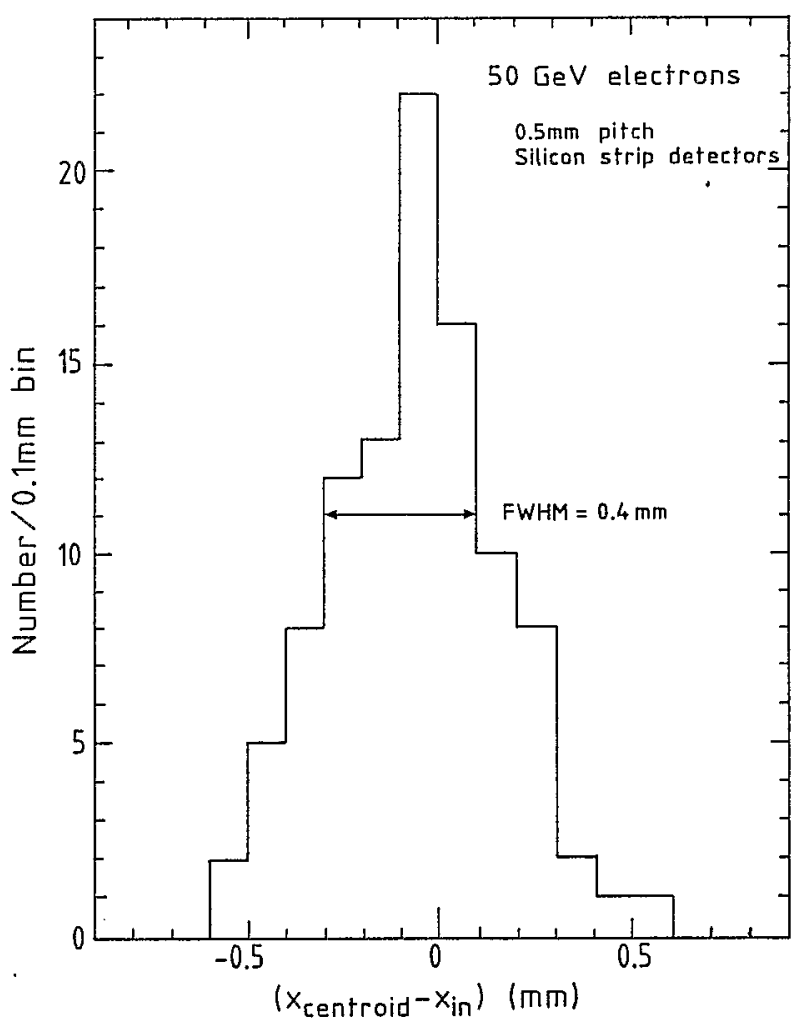

Fig. 3 Electron position accuracy at $5 \mathrm{X}_{0}$ sampling depth using $500 \mu \mathrm{m}$ pitch silicon detectors. Comparison of the charge-weighted centroid with the actual input position for 100 showers from $50 \mathrm{GeV}$ electrons, using EGS [14].

defined than a $2 \mathrm{~cm}$ cell, especially in the first $10 \mathrm{X}_{0}$, the spatial resolution needs to be improved. We propose to include silicon strip detector layers at $(5,10$, and 20$) \mathrm{X}_{0}$ to give submillimetre spatial resolution on, for example, the electron position. This, together with a fine-grained TRD [13], should allow the observation of electrons within a dense particle environment. The electron angular resolution in this device will be $1 \mathrm{mrad}$, whilst a typical jet will have $50 \%$ of the energy flux in a cone angle of $50 \mathrm{mrad}$, carried by 5-10 leading particles [3]. Figure 3 shows an EGS [14] simulation of the position error on $50 \mathrm{GeV}$ electrons in one projection at the $5 \mathrm{X}_{0}$ depth sampling plane. The FWHM is $0.4 \mathrm{~mm}$, so this method of isolating electrons seems to be quite promising. The SICAPO Collaboration is carrying out sóme experimental studies along these lines for a HERA detector. Note that these sampling planes considerably reduce the effective cell size of the electromagnetic section. Rather than calculate $\delta \eta \delta \phi$ from the $2 \mathrm{~cm} \times 2 \mathrm{~cm}$ segmentation, the two-shower separation area should be used. For electrons of energies above $50 \mathrm{GeV}, 90 \%$ of the shower energy is contained within $\pm 2 \mathrm{~mm}$, according to the EGS. Thus, taking $5 \mathrm{~mm}$ as a conservative two-shower separation distance, the electromagnetic section has an effective segmentation of $0.02 \times$ $1.5^{\circ}$, rather than $0.08 \times 6^{\circ}$ for the full $2 \mathrm{~cm} \times 2 \mathrm{~cm}$ area.

\subsection{Hadronic section}

The hadron segmentation is $2 \mathrm{~cm} \times 2 \mathrm{~cm} \times 0.5 \lambda$ for the first six absorption lengths, then $10 \mathrm{~cm} \times 10 \mathrm{~cm} \times$ $1 \lambda$ for three additional absorption lengths. The silicon section is followed by an $\mathrm{Fe} /$ scint. tail-catcher of magnetized iron that also serves as the first part of the muon detector. This fine segmentation is unusual but very advantageous for a small-radius device, and circumvents the complication of building a projective device. With fine-grained longitudinal and lateral sampling, the energy-weighted centroid of the shower at each cell is readily calculated and the shower direction defined. Results of a simulation will be presented later. 
In a U/Si calorimeter the additional electronics costs of making fine segmentation are small compared with the cost of the silicon detectors themselves; but the pattern recognition benefits are enormous, as demonstrated in Section 6. For this detector the segmentation in Table 2 gives $\delta \eta \delta \phi=0.05 \times 3^{\circ}$ after the first hadronic sampling section $(\mathrm{r}=41 \mathrm{~cm}$, depth $=1.12 \lambda)$.

\subsection{Costs}

Although it is premature to estimate the overall cost with any degree of reliability, we want to ensure that the proposed device is not 'obviously prohibitively expensive'. To estimate this cost, we summarize the components in Table 3. The amounts given in Table 4 include those for the silicon detectors, cables, and readout, as far as the anticipated analog pipeline out to the trigger/readout electronics. They do not include any digitization cost nor the

\section{Table 3}

Components in the barrel region of the Si calorimeter.

(The end caps increase these requirements by $50 \%$.)

\begin{tabular}{|l|l|l|}
\cline { 2 - 3 } \multicolumn{1}{c|}{} & Electromagnetic section & Hadronic section \\
\hline Inner radius & $20 \mathrm{~cm}$ & $34 \mathrm{~cm}$ \\
Outer radius & $34 \mathrm{~cm}$ & $129 \mathrm{~cm}$ \\
Silicon area: & $82 \mathrm{~m}^{2}$ & $1320 \mathrm{~m}^{2}$ \\
readout channels & 26,000 & 158,000 \\
microplex chips & 204 & 1250 \\
SSD area: & $10 \mathrm{~m}^{2}$ & $11.5 \mathrm{~m}^{2}$ \\
readout channels & 15,000 & 18,500 \\
microplex chips & 120 & 150 \\
\hline
\end{tabular}

Table 4

Cost of the Si calorimeter

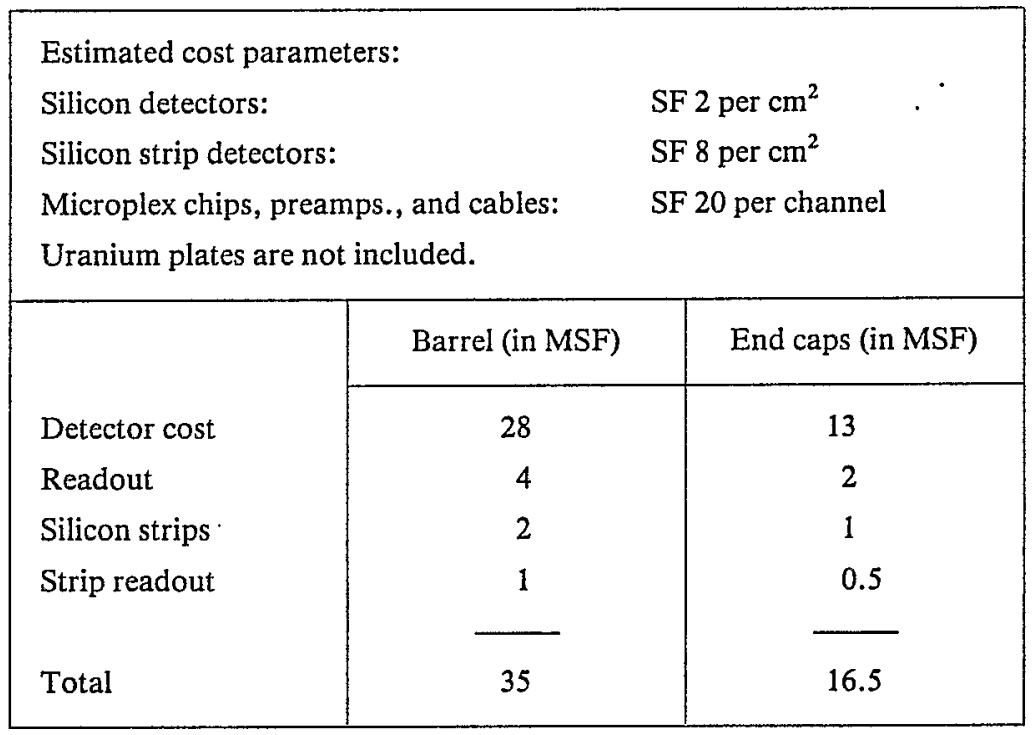


cost of the uranium plates. The assumption is that the cost of the Si detector for COSICA will decrease from the $\mathrm{SF} 6$ per $\mathrm{cm}^{2}$ at present being paid for the SICAPO devices, to SF 2 per $\mathrm{cm}^{2}$. This reduction assumes that the cost of the bulk silicon is reduced as well. For the readout we use microplex chips $[15,16]$ at SF 20 per channel, including cabling and on-board radiation-hardened preamplifiers to drive $2 \mathrm{~m}$ of cable out to the microplex chips. As cited in Table 3 , this detector requires $2100 \mathrm{~m}^{2}$ of silicon detectors. This is a factor of $40-60$ more than the overall needs of current projects. Clearly, good industrial engineering techniques will be needed, but large-scale production of semiconductors is quite usual nowadays. The investment in silicon is MSF 41 for pad detectors, and a further MSF 3 for silicon strip detectors. The 326,000 readout channels take 2580 microplex chips. Costs are estimated at MSF 7.

\subsection{Radiation damage}

Can the silicon survive just outside this enormous radiation source? Whilst there is much experience of radiation damage to silicon, very little of it is relevant to calorimetry. Most studies have concentrated on the increase in leakage current under radiation dose. The majority of experiments have used fully depleted detectors made with high-resistivity silicon - the damage is worse, the higher the resistivity.

The only studies that measured the energy resolution of a detector before and after radiation exposure were done at CERN. Two detectors, one of $3.7 \mathrm{k} \Omega \cdot \mathrm{cm}$ and the other of $9 \mathrm{k} \Omega \cdot \mathrm{cm}$ resistivity, were exposed. The first survived a dose of $0.7 \times 10^{3} \mathrm{~Gy}$ with little effect; the other became unusable for calorimetry at a dose of $10^{4} \mathrm{~Gy}$ [17]. A similar, earlier exposure of a low-resistivity device indicated a safe operating dose for calorimetry of at least $2.5 \times 10^{3}$ Gy [18].

The radiation dose for COSICA has been simulated using the CERN radiation safety program FLUKA [19]. The procedure is described in Section 5, and gives the dose corresponding to $10^{7} \mathrm{~s}$ of operation at a luminosity of $10^{33} \mathrm{~cm}^{-2} \mathrm{~s}^{-1}$, or one machine-year of good operation. As one would expect, the end caps receive the highest dose. The limiting dose of $2.5 \times 10^{3} \mathrm{~Gy}$ in the end caps was reached at $\theta=7^{\circ}$. Translating this into the apparatus shown in Fig. 2, the highest radiation dose is given to the corners of the two tilted segments closest to the beam. For this design, the dose at these corners is less than the fatal limit. Most of the detector receives a much lower dose. Therefore, any radiation damage will be highly local, and good monitoring, using radioactive sources placed on the detector and monitored during 'dump and fill' periods, will give a continual record of the resolution of the various detector segments. In this way any damage can be caught during the early stages and a replacement scheme can be implemented for a few detectors.

There is still one unknown factor. In a U/Si calorimeter, each high-energy ( $>100 \mathrm{GeV}$ ) particle generates several thousand low-energy neutrons that form a 'gas' percolating through the detector for distances of the order ' of several interaction lengths. One of these neutrons may traverse many silicon layers. Neutrons of energy as low as $1 \mathrm{keV}$ can produce dislocations in the silicon lattice. Therefore there may be enhanced radiation damage, which is not included in the FLUKA model. It is vital that this effect is looked into experimentally as part of an overall study of the use of silicon detectors for hadron calorimetry.

\subsection{Calorimeter performance}

We described the calorimeter Monte Carlo used in Section 3. This program was used to simulate this calorimeter's response to jets from the decay sequence $\mathrm{H} \rightarrow \mathrm{W}^{+}+\mathrm{W}^{-} \rightarrow 4$ jets, where the Higgs is produced at rest. Higgs masses of $0.6-1.0 \mathrm{TeV}$ were used, and $\mathrm{W}$ angles of $45^{\circ}$ and $90^{\circ}$ from the beams were compared. The simulation, described in Section 6, gives a resolution of $8.5 \%$ in the W mass for $90^{\circ}$ incidence, so the compact nature of this calorimeter does not cause any problems when making a very good reconstruction of high-energy $W$ decays. As the polar angle of the $\mathrm{W}$ is decreased, the resolution worsens somewhat because of the non-projective

geometry. However, the effects are not large and can be reduced by a more sophisticated jet algorithm. The simulation shows that this device will do an excellent job of jet separation and reconstruction. 
This detector has other useful features for LHC physics. We propose to insert three additional silicon strip layers in the hadronic calorimeter. One use for these layers is to look for non-showering tracks that stay, for two interaction lengths, within the trajectory limited by multiple Coulomb scattering. This would give an early indication of a muon and may be useful for trigger schemes. Another, more speculative, application of these layers could be that of giving greatly enhanced position information about jets. In the experimental study of hadron shower development in uranium [20], it was noticed that the total ionization peak, monitored by dosimeters, was very narrow - only a few millimetres FWHM. The fission product distribution, on the other hand, had a width characteristic of an interaction length. This suggests that the electromagnetic component of high-energy showers, carrying typically $50 \%$ of the incident energy for high-energy particles, remains tightly collimated around the incident particle direction. Therefore, using the charge-weighted centroid in silicon strip layers may give a very good measure of the leading particles in a quark or gluon jet. This idea must be explored experimentally to see if it is a useful property, or if fluctuations will obliterate any improvement over the trajectory information from the full pad structure. However, if this possibility is borne out, then the jet resolution may be significantly improved.

A complete calorimeter layout, including non-silicon-readout end-cap coverage at $\theta<7^{\circ}$, a magnet coil, and muon coverage ( $99 \%$ of $4 \pi$ ), is shown in Fig. 2. The end-cap coverage down to 10 mrad will pick up quark jets close to the beam as an additional tag on the production of, for example, Higgs through WW fusion - the quarks, which radiate the W's forming the Higgs, receive a transverse-momentum kick. The radiation level prohibits the use of silicon as a readout medium in this region. However, other media could be considered, e.g. circulating liquid scintillator, or simple scintillator modules that could easily be changed. Thus the prospects should be good for making a 'missing total energy' trigger for some new physics studies, and the calorimetric coverage could be completed by extending the calorimetry all along the beam pipe. This is discussed in Section 7.

\subsection{Conclusion}

A set-up using a compact calorimeter with microvertex and lepton tagging detectors seems to be capable of doing much of the new physics to be explored in the TeV mass region. The calorimetry is of very high quality. The idea of subdividing the cells with additional silicon devices may give even better angle and position information. There remain some important questions about radiation tolerance which should be explored with a well-planned and well-funded experimental program aimed at making a prototype U/Si hadronic calorimeter within the next few years.

\section{DOSE TO THE U/Si CALORIMETER}

\subsection{The calculation}

The event generator used to simulate the pp interactions was PYTHIA of the Lund model [21]. A file of 44,000 secondaries from 250 events at $10+10 \mathrm{TeV}$ was produced. The file was randomized before being used in the subsequent analysis. Particles from this file were used as input to the Monte Carlo Cascade Program FLUKA [19]. The PYTHIA input file was not subdivided into batches, i.e. no statistical errors were obtained. Leading-particle biasing was used in FLUKA and EGS4.

The geometries used to simulate the calorimeter structures are illustrated in Figs. 4 and 5 . For the lateral calculation, the calorimeter (Fig. 4), was represented by eight pairs of concentric cylinders of uranium ( $3 \mathrm{~mm}$ thick) and aluminium ( $1 \mathrm{~mm}$ thick). Aluminium was used instead of silicon since a PEGS data file for use by the EGS at collider energies was not available at the time of the calculation. An inner radius of $20 \mathrm{~cm}$ was assumed, and the half-length of the calorimeter was taken to be $120 \mathrm{~cm}$, divided into $10 \mathrm{~cm}$ long bins.

The forward calorimeter, illustrated in Fig. 5, was simulated by 11 pairs of discs composed of $3 \mathrm{~mm}$ thick uranium and $1 \mathrm{~mm}$ aluminium (outer radius $25 \mathrm{~cm}$ ), placed perpendicularly to the beam direction, starting at $115 \mathrm{~cm}$ from the interaction point. The vacuum chamber was represented by an empty cylinder of $2 \mathrm{~cm}$ radius passing through the calorimeter structure. 


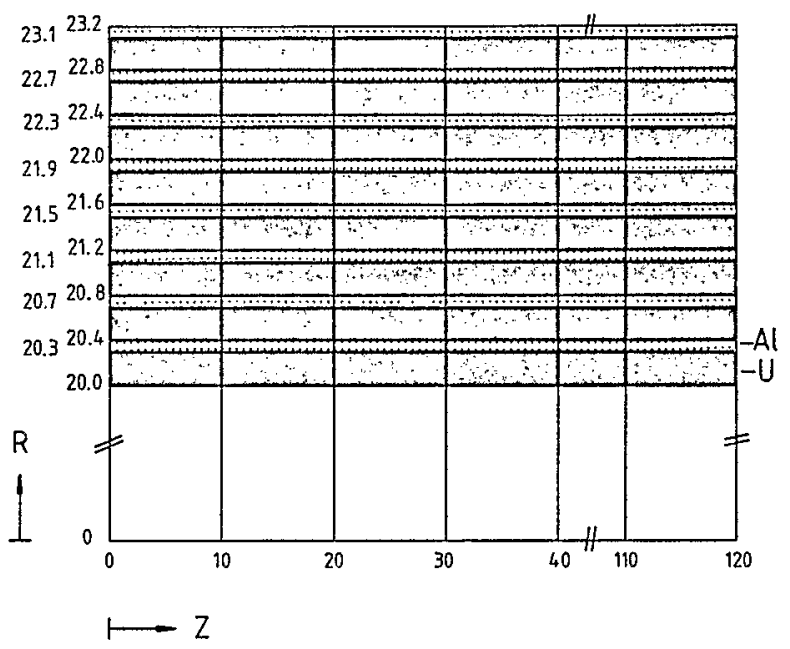

Fig. 4 Geometry for the lateral calorimeter simulation. All dimensions are given in centimetres.

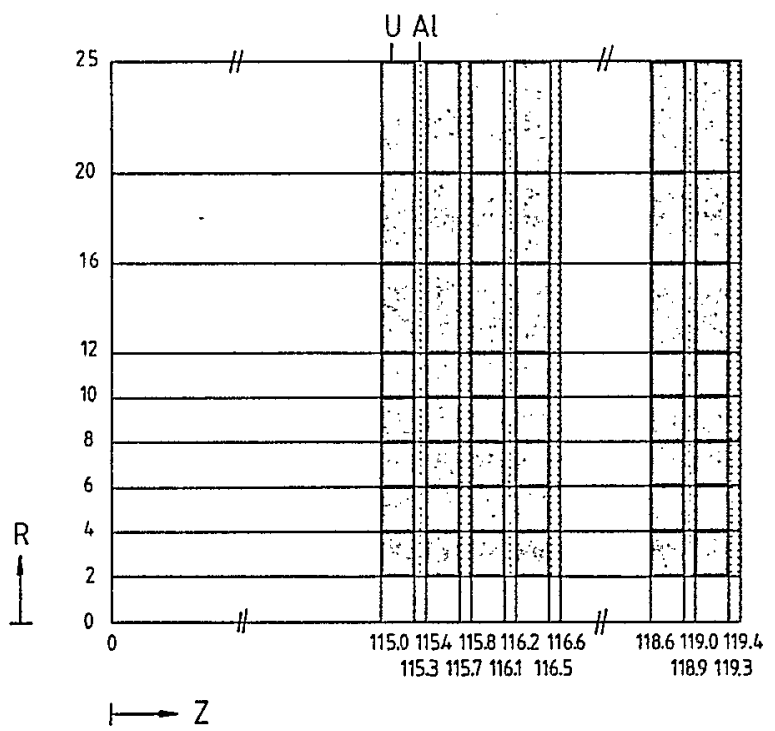

Fig. 5 Geometry for the forward calorimeter simulation. All dimensions are given in centimetres.

The value of the energy deposited in different parts of the aluminium structure was stored in the FLUKA calculation.

\subsection{Results and conclusions}

The estimated dose per year in the aluminium plates, based on an integrated luminosity of $10^{40} \mathrm{~cm}^{-2}$, is given in Tables $5 \mathrm{a}$ and $5 \mathrm{~b}$. These tables give both the total dose and the part deposited by electrons in the electromagnetic showers coming from incident photons and the decay of produced $\pi^{0}$ mesons. In the barrel the maximum dose occurs in the second layer of aluminium in the $40-50 \mathrm{~cm}$ bin downstream of the interaction point: the value of the yearly integrated dose is $8 \times 10^{3} \mathrm{~Gy}$. Note that in the calculations described here, neither the extra energy due to photons from fission in the uranium nor the energy deposition by fission-produced neutrons is considered. To account for this, calculations using the HETC [22] code coupled to the neutron transport code MORSE [23] must 
Table 5a

Annual dose from the lateral calculation (Gy/y)

\begin{tabular}{|c|c|c|c|c|c|c|c|}
\hline \multirow{2}{*}{$\mathrm{z}(\mathrm{cm})$} & \multicolumn{7}{|c|}{ Radius (cm) } \\
\hline & $20.3-20.4$ & $20.7-20.8$ & $21.1-21.2$ & $21.5-21.6$ & $21.9-22.0$ & $22.3-22.4$ & $22.7-22.8$ \\
\hline & \multicolumn{7}{|c|}{ Total energy density (dose) } \\
\hline $0-10$ & $1.7 \times 10^{3}$ & $3.2 \times 10^{3}$ & $2.7 \times 10^{3}$ & $2.8 \times 10^{3}$ & $2.9 \times 10^{3}$ & $1.7 \times 10^{3}$ & $1.4 \times 10^{3}$ \\
\hline $10-20$ & $2.6 \times 10^{3}$ & $2.9 \times 10^{3}$ & $2.9 \times 10^{3}$ & $1.9 \times 10^{3}$ & $1.6 \times 10^{3}$ & $2.9 \times 10^{3}$ & $9.9 \times 10^{2}$ \\
\hline $20-30$ & $3.6 \times 10^{3}$ & $2.7 \times 10^{3}$ & $2.3 \times 10^{3}$ & $2.1 \times 10^{3}$ & $1.4 \times 10^{3}$ & $1.4 \times 10^{3}$ & $9.6 \times: 10^{2}$ \\
\hline $30-40$ & $2.6 \times 10^{3}$ & $4.7 \times 10^{3}$ & $2.2 \times 10^{3}$ & $1.2 \times 10^{3}$ & $1.5 \times 10^{3}$ & $1.1 \times 10^{3}$ & $8.9 \times 10^{2}$ \\
\hline $40-50$ & $6.9 \times 10^{3}$ & $7.9 \times 10^{3}$ & $2.8 \times 10^{3}$ & $2.1 \times 10^{3}$ & $1.1 \times 10^{3}$ & $1.6 \times 10^{3}$ & $1.8 \times 10^{3}$ \\
\hline $50-60$ & $5.6 \times 10^{3}$ & $6.8 \times 10^{3}$ & $2.0 \times 10^{3}$ & $1.7 \times 10^{3}$ & $8.7 \times 10^{2}$ & $1.1 \times 10^{3}$ & $1.3 \times 10^{3}$ \\
\hline $60-70$ & $5.4 \times 10^{3}$ & $5.1 \times 10^{3}$ & $2.9 \times 10^{3}$ & $1.1 \times 10^{3}$ & $1.3 \times 10^{3}$ & $4.1 \times 10^{3}$ & $1.1 \times 10^{3}$ \\
\hline $70-80$ & $5.2 \times 10^{3}$ & $3.9 \times 10^{3}$ & $2.4 \times 10^{3}$ & $1.4 \times 10^{3}$ & $2.9 \times 10^{3}$ & $9.5 \times 10^{2}$ & $1.3 \times 10^{3}$ \\
\hline $80-90$ & $4.8 \times 10^{3}$ & $3.2 \times 10^{3}$ & $2.6 \times 10^{3}$ & $1.3 \times 10^{3}$ & $1.0 \times 10^{3}$ & $7.8 \times 10^{2}$ & $9.2 \times 10^{2}$ \\
\hline $90-100$ & $7.0 \times 10^{3}$ & $3.8 \times 10^{3}$ & $2.3 \times 10^{3}$ & $1.6 \times 10^{3}$ & $1.3 \times 10^{3}$ & $3.2 \times 10^{3}$ & $1.0 \times 10^{3}$ \\
\hline $100-110$ & $6.5 \times 10^{3}$ & $1.7 \times 10^{3}$ & $3.0 \times 10^{3}$ & $2.1 \times 10^{3}$ & $9.6 \times 10^{2}$ & $6.1 \times 10^{2}$ & $1.1 \times 10^{3}$ \\
\hline $110-120$ & $2.0 \times 10^{4}$ & $1.0 \times 10^{4}$ & $2.2 \times 10^{3}$ & $1.5 \times 10^{3}$ & $1.0 \times 10^{3}$ & $3.3 \times 10^{3}$ & $1.0 \times 10^{3}$ \\
\hline \multicolumn{8}{|c|}{ e.m. energy density (dose) } \\
\hline $0-10$ & $9.8 \times 10^{2}$ & $2.4 \times 10^{3}$ & $2.0 \times 10^{3}$ & $1.6 \times 10^{3}$ & $2.0 \times 10^{3}$ & $9.7 \times 10^{2}$ & $6.1 \times 10^{2}$ \\
\hline $10-20$ & $1.5 \times 10^{3}$ & $1.9 \times 10^{3}$ & $2.0 \times 10^{3}$ & $1.3 \times 10^{3}$ & $9.2 \times 10^{2}$ & $1.5 \times 10^{3}$ & $4.6 \times 10^{2}$ \\
\hline $20-30$ & $2.2 \times 10^{3}$ & $1.5 \times 10^{3}$ & $1.2 \times 10^{3}$ & $1.2 \times 10^{3}$ & $7.6 \times 10^{2}$ & $7.3 \times 10^{2}$ & $3.8 \times 10^{2}$ \\
\hline $30-40$ & $1.5 \times 10^{3}$ & $2.6 \times 10^{3}$ & $1.3 \times 10^{3}$ & $5.8 \times 10^{2}$ & $4.7 \times 10^{2}$ & $2.9 \times 10^{2}$ & $1.0 \times 10^{2}$ \\
\hline $40-50$ & $5.6 \times 10^{3}$ & $6.4 \times 10^{3}$ & $1.6 \times 10^{3}$ & $1.0 \times 10^{3}$ & $2.1 \times 10^{2}$ & $7.4 \times 10^{2}$ & $9.3 \times 10^{1}$ \\
\hline $50-60$ & $3.9 \times 10^{3}$ & $5.4 \times 10^{3}$ & $1.2 \times 10^{3}$ & $6.2 \times 10^{2}$ & $2.7 \times 10^{2}$ & $2.4 \times 10^{2}$ & $4.5 \times 10^{2}$ \\
\hline $60-70$ & $3.5 \times 10^{3}$ & $3.7 \times 10^{3}$ & $1.6 \times 10^{3}$ & $2.8 \times 10^{2}$ & $3.7 \times 10^{2}$ & $3.3 \times 10^{2}$ & $1.2 \times 10^{2}$ \\
\hline $70-80$ & $3.6 \times 10^{3}$ & $1.7 \times 10^{3}$ & $1.0 \times 10^{3}$ & $4.6 \times 10^{2}$ & $1.3 \times 10^{2}$ & $8.8 \times 10^{1}$ & $7.2 \times 10^{1}$ \\
\hline $80-90$ & $3.8 \times 10^{3}$ & $1.5 \times 10^{3}$ & $1.3 \times 10^{3}$ & $1.3 \times 10^{2}$ & $2.3 \times 10^{2}$ & $1.0 \times 10^{2}$ & $6.2 \times 10^{1}$ \\
\hline $90-100$ & $3.3 \times 10^{3}$ & $1.1 \times 10^{3}$ & $1.9 \times 10^{2}$ & $1.6 \times 10^{2}$ & $1.3 \times 10^{2}$ & $1.4 \times 10^{3}$ & $1.6 \times 10^{2}$ \\
\hline $100-110$ & $5.0 \times 10^{3}$ & $7.5 \times 10^{2}$ & $8.3 \times 10^{2}$ & $6.3 \times 10^{2}$ & $1.7 \times 10^{2}$ & $8.3 \times 10^{1}$ & $2.2 \times 10^{2}$ \\
\hline $110-120$ & $1.8 \times 10^{4}$ & $7.6 \times 10^{3}$ & $3.8 \times 10^{2}$ & $6.0 \times 10^{2}$ & $1.3 \times 10^{2}$ & $7.2 \times 10^{2}$ & $2.7 \times 10^{2}$ \\
\hline
\end{tabular}

eventually be made. It should also be realized that dose levels in the range $10^{4}$ to $10^{5} \mathrm{~Gy}$ are sufficient to render useless most active solid-state electronic circuits [24, 25], to darken optical fibres [26] and plastic scintillators [27] and to cause damage to silicon radiation-detectors [12].

In the forward case, an annual dose of $10^{4} \mathrm{~Gy}$ would be exceeded at radii of less than $20 \mathrm{~cm} ; 10^{5} \mathrm{~Gy}$ would be exceeded at radii of less than $8 \mathrm{~cm}$. Again, caution with regard to the neglect of fissioning in these calculations needs to be expressed: it is estimated that this could double the dose levels described here, but the damage level in a silicon detector could be increased by an additional factor owing to the increased sensitivity of the detector to fission neutrons. 
Table 5b

Annual dose from the forward calculation (Gy/y)

\begin{tabular}{|c|c|c|c|c|c|c|c|}
\hline \multirow{3}{*}{$\mathrm{z}(\mathrm{cm})$} & \multicolumn{7}{|c|}{ Radius in $(\mathrm{cm})$} \\
\hline & $2-4$ & $4-6$ & $6-8$ & $8-10$ & $10-12$ & $12-16$ & $16-20$ \\
\hline & \multicolumn{7}{|c|}{ Total energy density (dose) } \\
\hline$|15.3-115.4|$ & $1.9 \times 10^{5}$ & $7.6 \times 10^{4}$ & $2.9 \times 10^{4}$ & $2.8 \times 10^{4}$ & $8.1 \times 10^{3}$ & $7.7 \times 10^{3}$ & $3.9 \times 10^{3}$ \\
\hline $\mid 15.7-115.8$ & $4.9 \times 10^{5}$ & $2.2 \times 10^{5}$ & $4.9 \times 10^{4}$ & $2.8 \times 10^{4}$ & $1.8 \times 10^{4}$ & $1.2 \times 10^{4}$ & $6.4 \times 10^{3}$ \\
\hline $116.1-116.2$ & $8.4 \times 10^{5}$ & $2.2 \times 10^{5}$ & $1.3 \times 10^{5}$ & $5.3 \times 10^{4}$ & $4.3 \times 10^{4}$ & $1.9 \times 10^{4}$ & $1.3 \times 10^{3}$ \\
\hline $116.5-116.6$ & $1.1 \times 10^{6}$ & $2.9 \times 10^{5}$ & $1.1 \times 10^{5}$ & $5.7 \times 10^{4}$ & $3.9 \times 10^{4}$ & $3.4 \times 10^{4}$ & $9.1 \times 10^{3}$ \\
\hline $116.9-117.0$ & $1.7 \times 10^{6}$ & $2.7 \times 10^{5}$ & $4.1 \times 10^{5}$ & $6.7 \times 10^{4}$ & $5.1 \times 10^{4}$ & $3.7 \times 10^{4}$ & $3.2 \times 10^{3}$ \\
\hline $117.3-117.4$ & $1.7 \times 10^{6}$ & $2.9 \times 10^{5}$ & $1.3 \times 10^{5}$ & $6.4 \times 10^{4}$ & $3.7 \times 10^{4}$ & $2.4 \times 10^{4}$ & $1.6 \times 10^{3}$ \\
\hline $\mid 17.7-117.8$ & $1.6 \times 10^{6}$ & $5.8 \times 10^{5}$ & $1.1 \times 10^{5}$ & $5.1 \times 10^{4}$ & $1.1 \times 10^{5}$ & $3.8 \times 10^{4}$ & $9.5 \times 10^{3}$ \\
\hline $118.1-118.2$ & $1.5 \times 10^{6}$ & $4.2 \times 10^{5}$ & $1.9 \times 10^{5}$ & $7.4 \times 10^{4}$ & $4.1 \times 10^{4}$ & $3.0 \times 10^{4}$ & $1.5 \times 10^{3}$ \\
\hline $118.5-118.6$ & $1.6 \times 10^{6}$ & $3.3 \times 10^{5}$ & $1.2 \times 10^{5}$ & $7.9 \times 10^{4}$ & $1.3 \times 10^{5}$ & $2.4 \times 10^{4}$ & $9.4 \times 10^{3}$ \\
\hline $\mid 18.9-119.0$ & $1.9 \times 10^{6}$ & $5.0 \times 10^{5}$ & $1.6 \times 10^{5}$ & $5.1 \times 10^{4}$ & $3.8 \times 10^{4}$ & $1.1 \times 10^{4}$ & $1.7 \times 10^{3}$ \\
\hline $\mid 19.3-119.4$ & $1.9 \times 10^{6}$ & $8.0 \times 10^{5}$ & $9.7 \times 10^{4}$ & $4.0 \times 10^{4}$ & $1.6 \times 10^{4}$ & $8.2 \times 10^{3}$ & $\times 10^{3}$ \\
\hline \multicolumn{8}{|c|}{ e.m. energy density (dose) } \\
\hline $115.3-115.4$ & $8.8 \times 10^{4}$ & $2.2 \times 10^{4}$ & $1.4 \times 10^{4}$ & $7.3 \times 10^{3}$ & $3.6 \times 10^{3}$ & $3.1 \times 10^{3}$ & $1.6 \times 10^{2}$ \\
\hline $115.7-115.8$ & $4.2 \times 10^{5}$ & $1.7 \times 10^{5}$ & $3.1 \times 10^{4}$ & $2.1 \times 10^{4}$ & $1.3 \times 10^{4}$ & $8.8 \times 10^{3}$ & $2.7 \times 10^{2}$ \\
\hline $116.1-116.2$ & $6.9 \times 10^{5}$ & $1.8 \times 10^{5}$ & $9.9 \times 10^{4}$ & $3.5 \times 10^{4}$ & $3.8 \times 10^{4}$ & $1.4 \times 10^{4}$ & $7.7 \times 10^{3}$ \\
\hline $116.5-116.6$ & $1.0 \times 10^{6}$ & $2.2 \times 10^{5}$ & $9.7 \times 10^{4}$ & $3.3 \times 10^{4}$ & $3.3 \times 10^{4}$ & $3.0 \times 10^{4}$ & $7.0 \times 10^{2}$ \\
\hline $116.9-117.0$ & $1.4 \times 10^{6}$ & $2.1 \times 10^{5}$ & $3.9 \times 10^{5}$ & $5.3 \times 10^{4}$ & $4.7 \times 10^{4}$ & $3.0 \times 10^{4}$ & $2.9 \times 10^{3}$ \\
\hline $117.3-117.4$ & $1.5 \times 10^{6}$ & $2.4 \times 10^{5}$ & $1.2 \times 10^{5}$ & $5.5 \times 10^{4}$ & $3.1 \times 10^{4}$ & $1.6 \times 10^{4}$ & $1.5 \times 10^{3}$ \\
\hline $117.7-117.8$ & $1.4 \times 10^{6}$ & $5.1 \times 10^{5}$ & $9.6 \times 10^{4}$ & $3.7 \times 10^{4}$ & $1.0 \times 10^{5}$ & $2.0 \times 10^{4}$ & $6.2 \times 10^{3}$ \\
\hline $118.1-118.2$ & $1.4 \times 10^{6}$ & $3.8 \times 10^{5}$ & $1.4 \times 10^{5}$ & $5.8 \times 10^{4}$ & $3.6 \times 10^{4}$ & $2.7 \times 10^{4}$ & $6.5 \times 10^{2}$ \\
\hline $118.5-118.6$ & $1.4 \times 10^{6}$ & $3.0 \times 10^{5}$ & $8.9 \times 10^{4}$ & $6.2 \times 10^{4}$ & $1.2 \times 10^{5}$ & $1.2 \times 10^{4}$ & $7.6 \times 10^{2}$ \\
\hline $118.9-119.0$ & $1.7 \times 10^{6}$ & $4.5 \times 10^{5}$ & $1.2 \times 10^{5}$ & $4.2 \times 10^{4}$ & $2.8 \times 10^{4}$ & $6.9 \times 10^{3}$ & $1.3 \times 10^{2}$ \\
\hline $119.3-119.4$ & $1.7 \times 10^{6}$ & $7.5 \times 10^{5}$ & $7.1 \times 10^{4}$ & $3.4 \times 10^{4}$ & $9.5 \times 10^{3}$ & $4.7 \times 10^{3}$ & $1.7 \times 10^{2}$ \\
\hline
\end{tabular}

\section{PERFORMANCE OF THE SMALL-RADIUS CALORIMETER}

\subsection{The test case}

The hadronic decay of W bosons originating from a heavy Higgs at rest has been used as a test case to study the behaviour of various calorimeter set-ups. The calorimeter has to perform the following tasks:

- recognize and resolve two-jet structures from W decays;

- identify the $\mathrm{W}$ by measuring its mass as precisely as possible;

- measure the energy and angle of the $W$.

W decays coming from a Higgs boson of $600 \mathrm{GeV}$ or $1000 \mathrm{GeV}$ mass produce jet pairs with a separation of typically $32^{\circ}$ or $17^{\circ}$, respectively. The task of resolving these jets is impeded by the following effects:

- gluon radiation (jet broadening); 
- fragmentation;

- spread of showers in the calorimeter.

All these effects have been taken into account in the following studies.

Technically a $\mathrm{W}$ boson $(\mathrm{m}=83 \mathrm{GeV})$ is first produced at rest and boosted according to the assumed Higgs mass. The $\mathrm{W}$ then decays isotropically into a quark-antiquark pair, which is then transferred into the jet final state by gluon radiation and fragmentation according to the Lund model [28]. Finally, the response of the calorimeter to the fragmentation products is simulated.

The calorimeter performance is measured using the following criteria:

- two-jet reconstruction efficiency;

- two-jet mass resolution;

- angular resolution (quality of opening-angle measurement).

\subsection{The calorimeter simulation}

\subsubsection{The calorimeter response}

The calorimeter response, simulated according to Section 3, affects both the angular resolution and the energy resolution of the generated jets. A correct simulation is therefore essential for realistic studies. The following list summarizes the parameters used:

- optimized longitudinal and transverse shower parametrization as described in Section 3.

- relative response to electrons and pions $\mathrm{e} / \pi=1.0$

- relative response to electrons and muons $\mu / \mathrm{e}=1.4$;

- relative electromagnetic resolution $\sigma(\mathrm{E}) / \mathrm{E}=15 \% / \sqrt{\mathrm{E}}$

- relative hadronic resolution $\sigma(\mathrm{E}) / \mathrm{E}=35 \% / \sqrt{\mathrm{E}}$.

\subsubsection{Calorimeter geometry}

The basic set-up for this study is a barrel with two sections of different longitudinal and transverse segmentation:

i) A fine-grained FRONT part

- to have a very good sampling of the complete electromagnetic fraction of the jet as well as of the main hadronic part;

- to provide the information for the jet pattern recognition and the two-jet separation power.

The total depth of the front part is 6 hadronic absorption lengths $\lambda$ in 12 consecutive samplings. The transverse sampling $T$ was varied as described in the results.

\section{ii) A coarser BACK part}

- to obtain a complete measurement of the hadronic-jet energy.

The depth of the back part is again 6 hadronic absorption lengths, but in only 3 samplings, with a relatively coarse separation in the transverse direction of $10 \mathrm{~cm} \times 10 \mathrm{~cm}$. As already mentioned, the main-jet separation power is expected to come from the front part. The back part will mainly provide the full measurement of hadronic energy.

Figure 6 illustrates the jet separation for a single event (W boson from a $600 \mathrm{GeV}$ Higgs) generated in the standard compact calorimeter with $20 \mathrm{~cm}$ inner radius and $2 \mathrm{~cm} \times 2 \mathrm{~cm}$ transverse sampling in the front part. Each Lego-plot represents a $100^{\circ} \times 100^{\circ}$ window in the 15 longitudinal layers of the calorimeter. The thick line divides the figure into the front part and the back part. The energy scale is different and arbitrary for each plot. The two-jet topology is clearly visible in the first layers and is then washed out by the spread of the hadronic shower component. It is evident that the early, and clearly separated, development of the two electromagnetic components is of great help for the pattern recognition. This plot motivates the chosen longitudinal structure described in this subsection. 


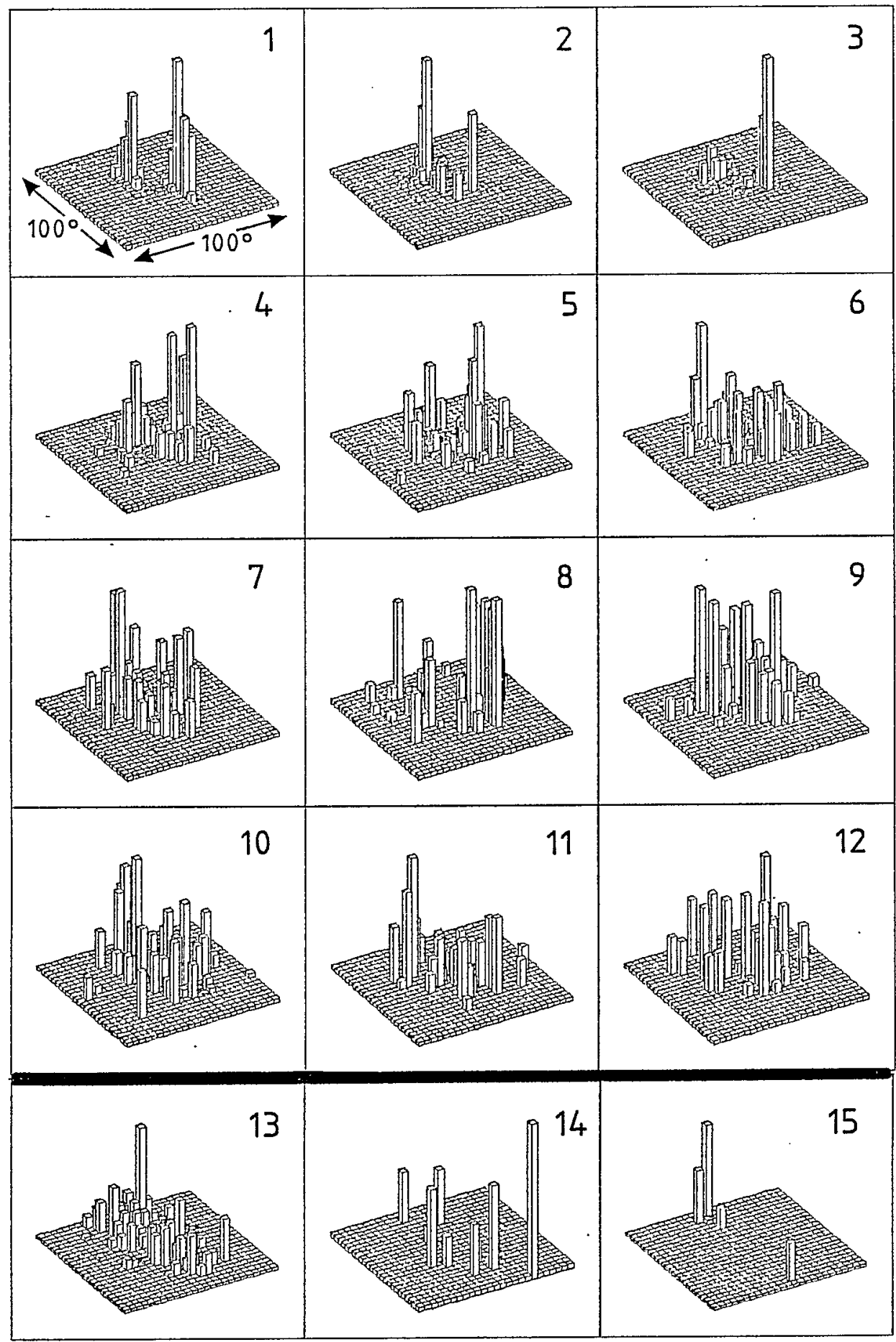

Fig. $6 \mathrm{~W}$ boson originating from a $600 \mathrm{GeV}$ Higgs producing a jet pair in the 15 longitudinal layers of the standard calorimeter

\subsection{Reconstruction techniques}

Fine-grained calorimeters can, in principle, reconstruct the $\mathrm{W}$ mass using directly the measured cell energies. Each cell is assumed to represent a particle with the energy given by the response of the cell and mass zero. However, this method encounters problems in the environment of hadron machines where the energy flow of the underlying event is added to the signal from the $\mathrm{W}$ decay products. A more practical approach is the following two-step procedure: 


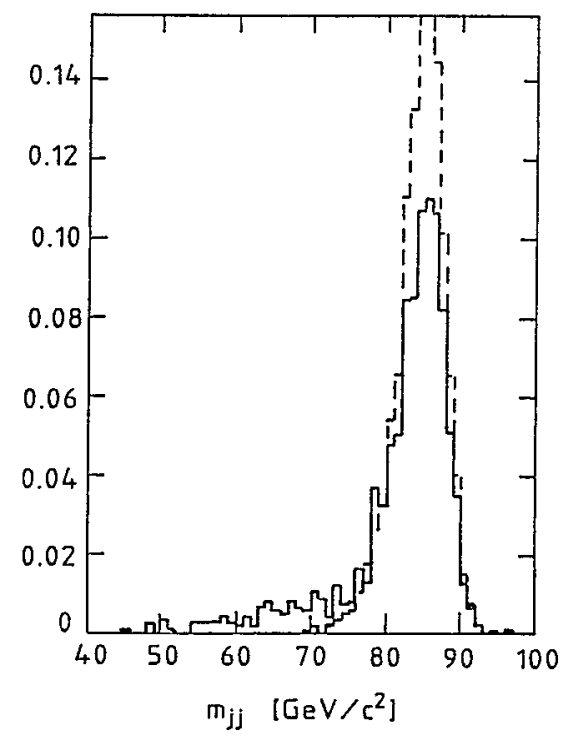

Fig. $7 \mathrm{~W}$ mass reconstruction using single cells (dashed line) and reconstructed clusters (full line)

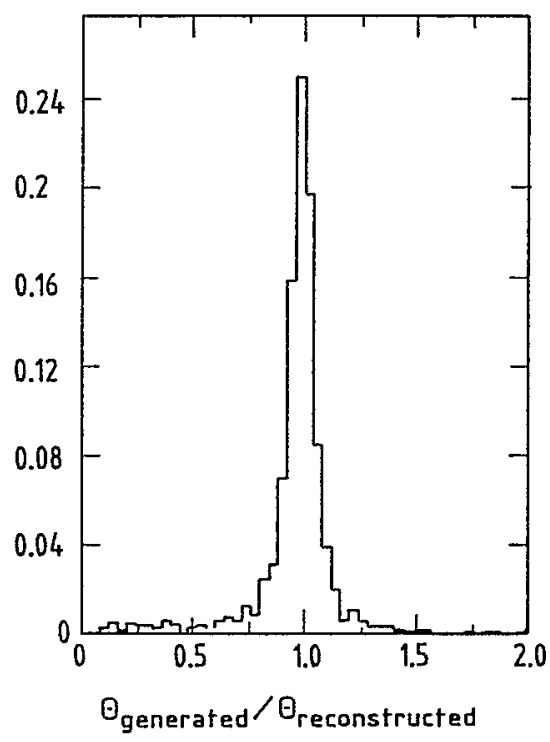

Fig. 8 Generated two-parton opening angle/observed two-jet opening angle

i) reconstruction of energy clusters (and possible energy correction techniques);

ii) calculation of the mass of the cluster system.

For this study the LUCLUS algorithm from the Lund JETSET package was used [29].

Figure 7 shows a comparison between the two approaches for the $\mathrm{W}$ mass reconstruction. The curves have been obtained for the compact calorimeter described in the results $(20 \mathrm{~cm}$ inner radius, $2 \mathrm{~cm} \times 2 \mathrm{~cm}$ transverse sampling in the front part). The W boson comes from a Higgs with a mass of $600 \mathrm{GeV}$. The dashed line shows the reconstructed mass using all cells in the calorimeter, including the energies of non-interacting particles (neutrinos and muons). The full curve is the result of the cluster reconstruction algorithm, as in a real experimental environment. The advantage of the cell method is evident ( $3.3 \mathrm{GeV}$ r.m.s. compared with $7.1 \mathrm{GeV}$ r.m.s. from the cluster method). The quality of the cluster reconstruction is demonstrated in Fig. 8 where the ratio between the generated two-parton opening angle and the observed two-jet opening angle is shown. The mean of the distribution is 0.96 with a r.m.s. spread of $17 \%$.

\subsection{Results}

This subsection summarizes the results on two-jet reconstruction efficiency $\epsilon$ and $W$ mass resolution obtained for a compact calorimeter set-up with an inner radius of $20 \mathrm{~cm}$.

\subsubsection{The effect of the transverse sampling in the front part}

The following defines the standard test case:

- transverse sampling $T$ in the front part $2 \mathrm{~cm} \times 2 \mathrm{~cm}$;

$-90^{\circ}$ incidence of the generated $\mathrm{W}$ with respect to the calorimeter surface;

- Higgs mass $600 \mathrm{GeV}$.

The performance obtained with this set-up (see the previous figures) is

- two-jet reconstruction efficiency, $\epsilon=87 \%$;

- W mass resolution, 7.1 GeV r.m.s.

Figure 9 shows the dependence of $\epsilon$ on the transverse sampling $T$ in the front part of the calorimeter in the extreme case of a $1000 \mathrm{GeV}$ Higgs. A rapid degradation of $\epsilon$ is observed when the transverse cell size increases beyond $5 \mathrm{~cm}$. 


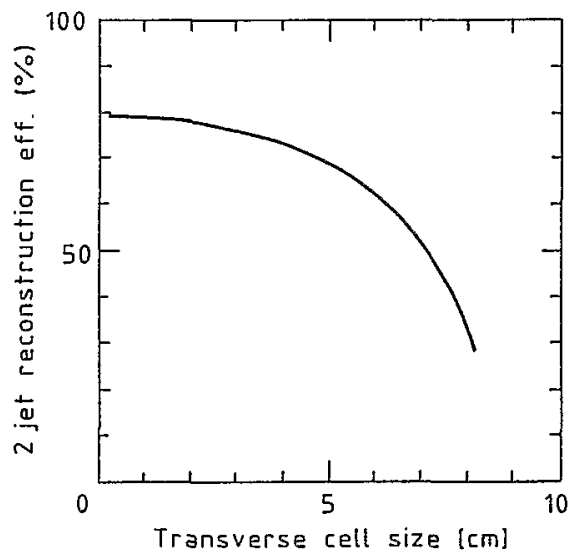

Fig. 9 Two-jet reconstruction efficiency versus transverse cell size in the front part of the calorimeter (Higgs mass $1000 \mathrm{GeV}$ )

This clearly demonstrates the need, for fine transverse sampling $($ e.g. $2 \mathrm{~cm})$ in the case of such a compact small-radius calorimeter.

\subsubsection{The effect of the boost}

The effect of the boost can be seen by comparing the case of a $600 \mathrm{GeV}$ Higgs decay, with that of a $1000 \mathrm{GeV}$ Higgs decay:

- $\epsilon$ decreases from $87 \%$ in the $600 \mathrm{GeV}$ case to $77 \%$ in the $1000 \mathrm{GeV}$ case;

- the mass resolution stays unchanged (7.1 GeV r.m.s. at $600 \mathrm{GeV}$ compared with $6.8 \mathrm{GeV}$ r.m.s. at $1000 \mathrm{GeV}$ ) for those cases where the cluster recognition was successful in finding two jets. The degraded angular resolution is compensated by the better energy resolution due to the higher boost of the fragmentation products.

\subsubsection{The effect of inclination}

Changing the $\mathrm{W}$ angle of incidence with respect to the calorimeter surface from $90^{\circ}$ to $45^{\circ}$ affects the calorimeter performance as follows:

- the two-jet separation probability $\epsilon$ is reduced from $87 \%$ to $77 \%$;

- the two-jet mass resolution is degraded from $7.1 \mathrm{GeV}$ r.m.s. to $8.4 \mathrm{GeV}$ r.m.s.

This can be explained by the non-projective barrel geometry of this calorimeter set-up. Inclined showers are smeared transversely and the effective longitudinal sampling is reduced.

\section{FORWARD CALORIMETER AND TOTAL ENERGY MEASUREMENT}

\subsection{Why forward calorimetry?}

Although these days one frequently speaks of 'hermetic' or $4 \pi$ calorimetry, the calorimetry does, in fact, have holes, at least for the beam pipes. The beam fragment jets are centred on these holes, with the result that generally a large fraction of the collision energy is undetected. For example, in the UAl calorimeters, which extend down to $\theta=0.2^{\circ}$, the total energy distribution peaks around $500 \mathrm{GeV}$ even for highly inelastic events (with $\mathrm{E}_{\mathrm{T}}=150 \mathrm{GeV}$ ) at $\sqrt{\mathrm{s}}=630 \mathrm{GeV}$. This loss is not surprising, as the probability of having a very energetic small-angle particle is high (all particles with $\mathrm{p}_{\mathrm{T}} \leq 300 \mathrm{MeV} / \mathrm{c}$ and $\mathrm{p}_{\mathrm{L}}>85 \mathrm{GeV} / \mathrm{c}$ will have $\theta<0.2^{\circ}$ ). To collect a similar fraction of the total energy at the much higher energy of the LHC, one would need to scale down the minimum angle by approximately the ratio of the beam energies, i.e. by $\approx 0.3 \mathrm{GeV} / 8 \mathrm{TeV}=0.04$ to $\approx 0.14 \mathrm{mrad}$. This is clearly not possible using normal techniques, with the superconducting low-beta quadrupoles and other machine elements starting at distances of $\approx 15$ to $20 \mathrm{~m}$ from the intersection $(0.14 \mathrm{mrad}$ at $20 \mathrm{~m}=2.8 \mathrm{~mm})$. Nevertheless, all 
secondary particles from an inelastic collision will eventually leave the pipe, which accepts only $\mathrm{x}_{\mathrm{F}}=1, \mathrm{pT}_{\mathrm{T}}=0$ positive particles, i.e. the non-interacting protons. In principle one could collect and measure all this energy by putting calorimeters in front of and in between the machine elements, and even, where necessary, calorimetrizing the machine elements themselves. We shall see later how this might be possible.

What would be the advantages of truly hermetic energy measurement? (Truly hermetic meaning that only non-interacting or small-t elastically scattered protons escape geometrically, and only muons, neutrinos, and other weakly interacting particles escape through the calorimeter.) The four examples given below come readily to mind.

\subsubsection{Counting the number of collisions or selecting single collisions}

For the higher-energy colliders we are forced, by the falling cross-sections $\left(\sigma \approx 1 / \mathrm{m}_{\mathrm{X}}^{2}\right)$, to go to very high luminosities. This means that we will usually have to work in conditions where 'pile-up' is common, i.e. more than one independent collision in the sensitive time of the detectors. Figure 10 is instructive; it shows the number of collisions per second which occur alone in a bunch crossing, as a function of luminosity. The conditions (solid line) are $t=25 \mathrm{~ns}$ between bunch crossings and $\sigma_{\text {inel }}=100 \mathrm{mb}$. The linear rise at low $\mathrm{L}$ turns into an exponential decrease above the 'optimum singles rate' which occurs when $\bar{n}=L \sigma t=1$. Because of this exponential decrease at

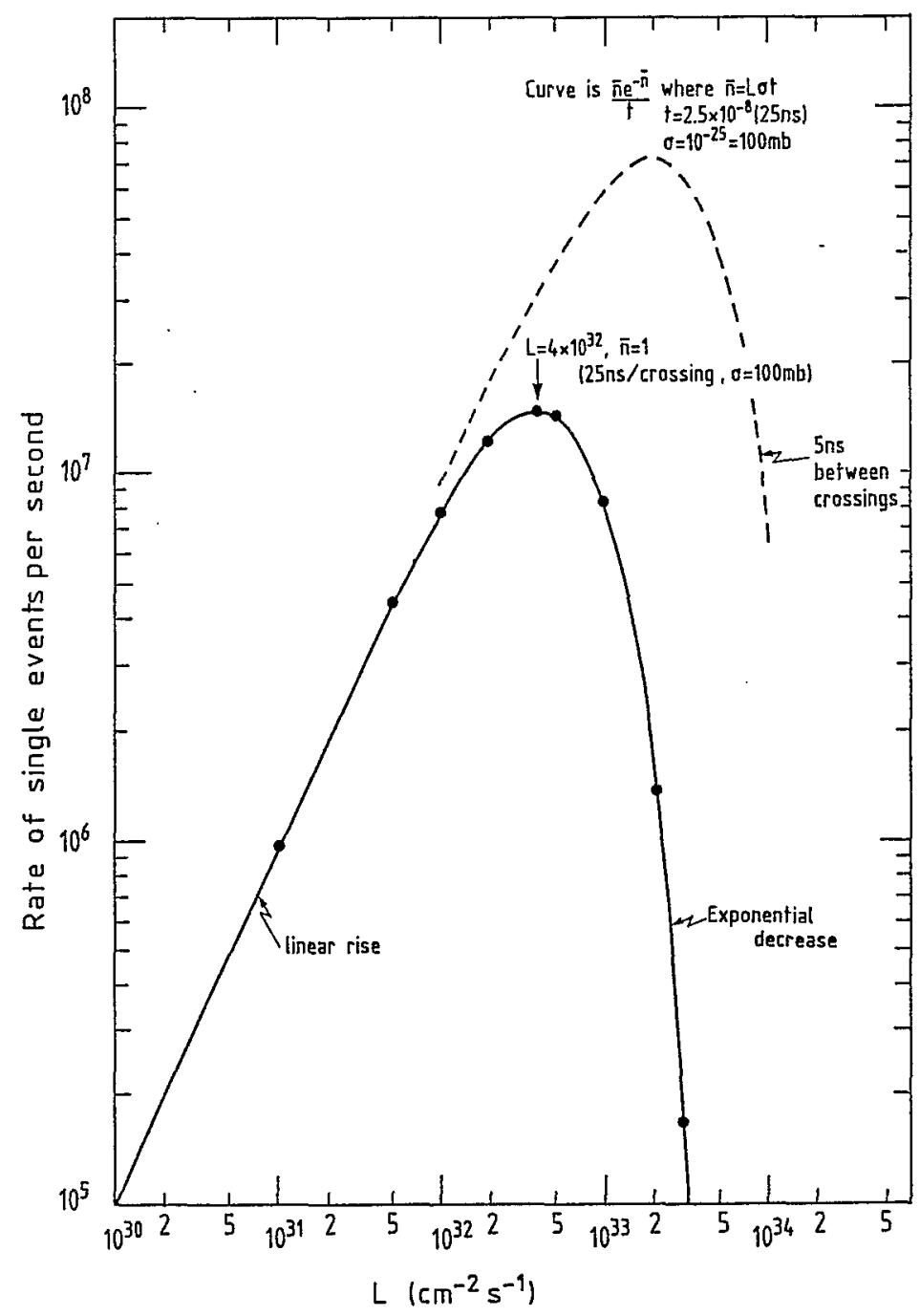

Fig. 10 Number of collisions per second which occur alone in a bunch crossing, as a function of luminosity 
very high luminosity $\left(\approx 10^{34} \mathrm{~cm}^{-2} \mathrm{~s}^{-1}\right.$ ), essentially every event will have one or more additional interactions in the same bunch crossing. Reducing the time between bunch crossings appears to help (see dashed line in Fig. 10 for $t=5 \mathrm{~ns}$ ), but of course this only holds true if the detectors are very fast - and calorimeters usually are not (not a few nanoseconds). Working above $L=4 \times 10^{32} \mathrm{~cm}^{-2} \mathrm{~s}^{-1}$ is therefore only efficient when searching for physics signatures which cannot be faked by pile-up (e.g., to have two balancing, very high-p $\mathrm{p}_{T}$ W's is fairly safe, but four jets which balance pairwise in $\mathrm{p}_{\mathrm{T}}$ is certainly not). For that class of physics which can be faked by double interactions, it is best to run close to or below $\bar{n}=1$ and to have a means of ensuring pile-up rejection. Total energy measurement and multiple vertex detection are two ways of doing this, and ideally one would have both. The 'beam jet' calorimetry can be rather coarse at these energies, because even a resolution $\delta \mathrm{E} / \mathrm{E}=100 \% / \sqrt{\mathrm{E}}$ is just over $1 \%$ at $8 \mathrm{TeV}$. Thus distinguishing one, two, three collisions should be very easy provided one can calorimetrize, even crudely, the elements around the downstream beam pipes (over a few hundred metres, preferably beyond the bend of the machine). We should repeat that we consider this to be of great importance for modest luminosities of $\approx 10^{32} \mathrm{~cm}^{-2} \mathrm{~s}^{-1}$, for physics which can be affected by pile-up, and where it is important to know that a single collision occurred. Multiple vertex recognition also helps but probably only off-line; it requires very good tracking along the beam direction, and it too fails at very high luminosity. For $\mathrm{L}=10^{34} \mathrm{~cm}^{-2} \mathrm{~s}^{-1}$, essentially every collision has another interaction within about $1 \mathrm{~mm}$ (assuming Gaussian bunches of length $\sigma_{\mathrm{x}} \approx 8 \mathrm{~cm}$ ).

\subsubsection{Studies of full rapidity structure of events}

Suppose we use the rule of thumb that within 3 units of rapidity of the beam particle there is the "beam fragmentation region', and that closer to $\theta=90^{\circ}$ there is the 'central region'. The boundary thus defined, which was at $\theta=33^{\circ}$ at the CERN Intersecting Storage Rings (ISR) $(\sqrt{\mathrm{s}}=63 \mathrm{GeV})$ and at $3.4^{\circ}$ at the p $\overline{\mathrm{p}}$ Collider, has shrunk to $\theta \approx 0.14^{\circ}$ at the $\mathrm{LHC}(5 \mathrm{~cm}$ at $20 \mathrm{~m})$. It will be very difficult to study physics in the beam fragmentation region at the LHC (this will be more easily done at the SSC provided long straight sections are built in). However, we may wish to know the full rapidity structure of an event - for example a rapidity gap of 3 units is a rather good signature for diffraction. Diffractive processes may be very interesting at LHC energies.

At the ISR, single diffractive excitation of protons extended up to about $10 \mathrm{GeV}$, and at the CERN p $\bar{p}$ Collider up to about $100 \mathrm{GeV}$. Using the relation $\mathrm{m}^{2} / \mathrm{s}=1-\mathrm{x}$ and the rule of thumb $\mathrm{x}_{\min }=0.975$, which gives the above two limits, we find $\mathrm{m}_{\max } \approx 2.5 \mathrm{TeV}$ at the LHC. It may be interesting to study these very massive 'diffractively excited' protons, and to do so one would like not only to detect the quasi-elastically scattered proton $\left(\mathrm{p}_{\mathrm{L}} \geq 0.975 \times 8000 \mathrm{GeV} / \mathrm{c}, \mathrm{p}_{\mathrm{T}} \leq 1 \mathrm{GeV} / \mathrm{c}\right)$, e.g. in silicon microstrip detectors inside the beam pipe, but also to be sure it was isolated in rapidity - no other particles below, say, $\theta \approx 0.14^{\circ}$.

\subsubsection{Missing-mass techniques}

The importance of sufficiently hermetic calorimetry to measure a 'missing transverse energy' vector, $\mathrm{E}_{\mathrm{T}}$ is now well established for W finding, SUSY searching and so forth. However, owing to the beam-pipe holes and the lack of very forward calorimetry, it was considered that trying to measure the 'missing longitudinal energy' $E_{L}$ would be hopeless. However, this could be so interesting that it is worth re-investigating. If our calorimetry is really $4 \pi$ apart from two small holes (say $\mathrm{p}_{\mathrm{L}} \geq 0.95 \mathrm{p}_{\mathrm{beam}}, \mathrm{p}_{\mathrm{T}} \leq 1 \mathrm{GeV} / \mathrm{c}$, positives) and has good resolution and granularity etc., we can measure missing mass: $h=\sqrt{ }\left[(\sqrt{s}-E)^{2}-E_{x}^{2}-E_{y}^{2}-E_{z}^{2}\right]$, where the $E_{i}$ 's are summed. Note that two conventional sources of $h$ and $E_{\mathrm{T}}$ at the p $\bar{p}$ Collider are: i) $\mathrm{W} \rightarrow \tau \nu, \tau \rightarrow$ hadron-jet; ii) $\mathrm{Z}+$ jet, $\mathrm{Z} \rightarrow \nu \bar{\nu}$. In both cases a hadron-jet recoils against nothing visible, but in case (i) $m \approx 0$ (the neutrino) and in case (ii) 向 $\approx \mathrm{m}_{Z}$. So even a missing-mass resolution of $\sigma \approx 25 \mathrm{GeV}$ would be very valuable for classifying these events and for searching for new phenomena, e.g. supersymmetry. We do not suppose that missing-mass resolutions of this order will be achievable at the $\mathrm{LHC}$, but even $\sigma(\mathrm{m})$ values of $200-400 \mathrm{GeV}$ are interesting in the case of $1 \mathrm{TeV}$ phenomena (e.g. $\left.Z^{\prime}\right)$. 


\subsubsection{Tagged parton collisions}

This possibility follows as a consequence of achievable missing-mass techniques, but here we consider the energy lost from the beam fragmentation regions whether or not it is visible in the central region. We add all the detected energy below $\theta_{\text {cut }}\left(\right.$ say $\left.0.2^{\circ}\right)$ on each side $\left(E_{L}, E_{R}\right)$ and trigger on events with, for example, $E_{L, R}<\left(E_{b e a m}-\right.$ $1 \mathrm{TeV}$ ) - this is effectively tagging interacting partons above $1 \mathrm{TeV}$, then studying the central region 'inclusively'.

The physics made possible by nearing the hermeticity required to realize the latter two techniques could result in a big payoff. If one could really trigger on events where a (say) $1.00 \pm 0.1 \mathrm{TeV}$ parton-parton collision has occurred, without any requirements on the final state of that collision, it would in some sense be analogous to the $\mathrm{e}^{+} \mathrm{e}^{-}$case. However, it is clear that this could only be achieved

i) for modest luminosities of $\approx 10^{32} \mathrm{~cm}^{-2} \mathrm{~s}^{-1}$ such that $\overline{\mathrm{n}} \leq 1$;

ii) by having sufficient forward calorimeter coverage so that it is extremely unlikely that a small-angle energetic hadron escapes detection (except 'elastic protons'). This probably means calorimetrizing beyond the machine bend, to catch leading neutrons.

\subsection{How to calorimetrize the fragmentation region}

A proper assessment of what might be achieved requires a 'realistic' model of the machine from the intersection region down to and round the bend, together with Monte Carlo generated $8 \mathrm{TeV}$ beam fragmentation jets. Then, taking into account both the materials and the magnetic fields, one could see just where the bulk of the energy is deposited. This has not yet been done. We understand that after $20 \mathrm{~m}$ of free space, a $40 \mathrm{~m}$ long quadrupole triplet is likely to be the first machine element. The front face of this element would obviously be about the hottest region of radiation (perhaps $=10^{4} \mathrm{~Gy} / \mathrm{y}$ close to the beam pipe), and this limits the choice of readout medium; liquid scintillator would be a possibility. [Drifting liquid calorimeters (liquid argon, TMP, etc.) are unlikely to be suitable, unless a very fast liquid can be found ( $<25 \mathrm{~ns}$ drift time), because the occupancy will be high-essentially every event will deposit energy here.] This quadrupole triplet can serve as an example of what may be done also with the other machine elements to minimize the energy deposited in inert material. It may be split into shorter, separated units (e.g. three separate quads) with the bore/vacuum pipe increased in diameter so that particles do not hit the pipe. Between these units, in gaps $\approx 1 \mathrm{~m}$ long, one inserts dense smaller-bore calorimetry - uranium or tungsten absorbers allow $6 \lambda-7 \lambda$ within $1 \mathrm{~m}$, including readout. Figure 11 sketches the
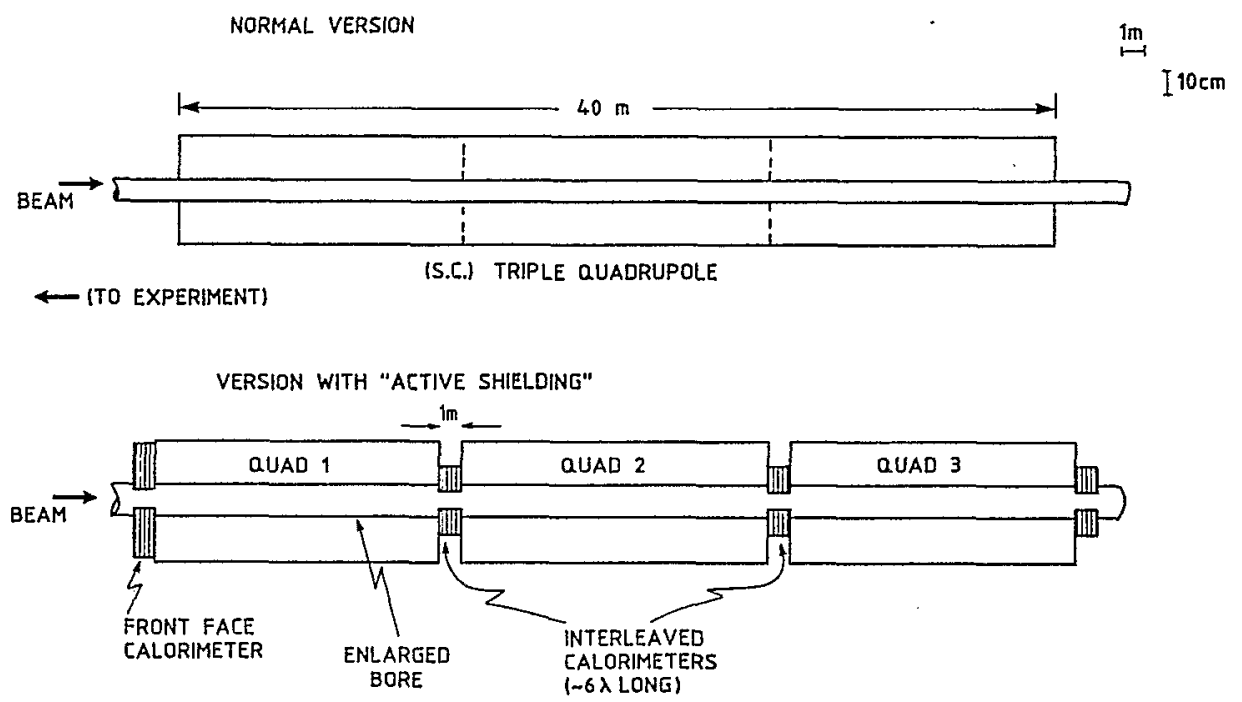

POSSIBLE WAY OF DETECTING "ALL" FORWARD ENERGY

Fig. 11 Active shielding of machine elements 


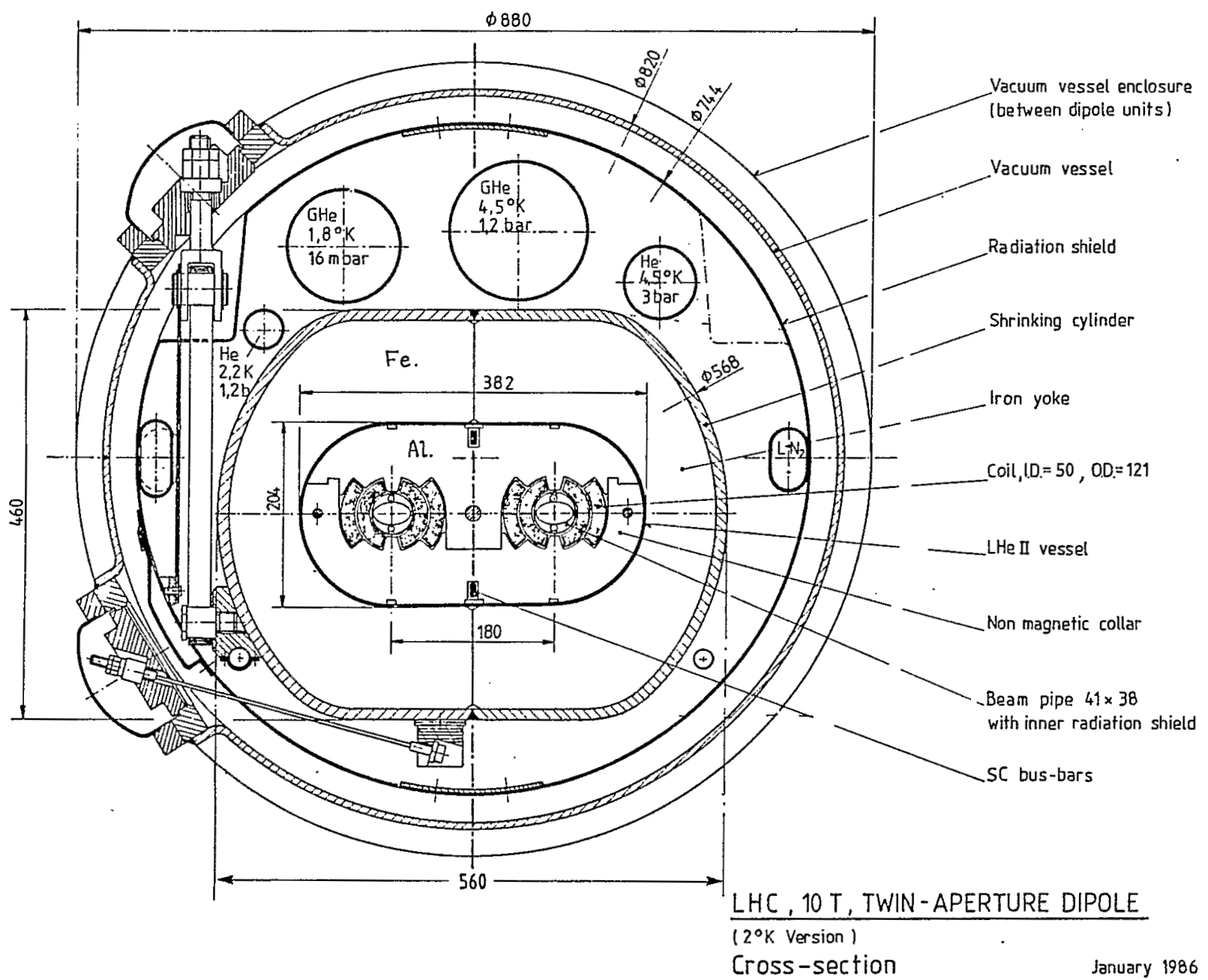

Fig. 12 Sketch of a typical superconducting machine twin-bore dipole

concept. In this way one attempts to preface all inert machine elements with 'active shielding'. Of course this is not ideal from the machine point of view: the magnets have somewhat larger bore and are shorter and more numerous - we will really be interleaving the experiment and the machine for the first $250 \mathrm{~m}$ or so. If this causes difficulties, another route may be possible, involving calorimetrizing the machine magnets themselves (an even more intimate interleaving). Figure 12 shows a sketch of a typical superconducting machine, twin-bore dipole. The only parts that seem really difficult to calorimetrize are the coils themselves, but at worst these are not more than 3-4 cm thick. The 'non-magnetic collar' surrounding these coils consists of a stack of laminated, (probably) aluminium plates, each $2 \mathrm{~mm}$ thick. This in turn is surrounded by iron laminations, and the whole is placed in a liquid-helium bath. It should be possible to design the magnet in such a way that every few centimetres (the radiation length of $\mathrm{Al}$ is $9 \mathrm{~cm}$, that of $\mathrm{Fe} 1.76 \mathrm{~cm}$ ) one plate is replaced by a detector layer. The requirements of the detector are that

- it operates at liquid-helium temperatures,

- has fast signals (25 $\mathrm{ns}$ ),

- is thin and compact,

- has the ability to extract signals with ease (1 thin wire or optical fibre?),

- is sufficiently radiation-hard so that it does not need to be replaced,

- it can take the strains when the magnetic field is switched on. 
These requirements are probably best met (except perhaps for the last point) by silicon wafers. We hope that experimental tests along these lines can be pursued, leading towards a prototype 'calorimetrized superconducting magnet'.

Clearly this is simply a presentation of some ideas, and much work is needed to assess their feasibility. However, we would urge the designers of the machine to maintain flexibility, and avoid finalizing the design before these studies have been made.

\section{CONCLUSIONS}

We have proposed a novel detector design for the LHC (SSC) machine - that of a compact silicon calorimeter. It has been shown that the small radius of this detector can be compensated by fine-grained lateral and longitudinal segmentation, and it is therefore realistic to consider a $4 \pi$ silicon calorimeter. The calorimeter would have uranium as absorber, and the $100 \mu \mathrm{m}$ polyethylene foils covering the silicon in the sandwich should bring $\mathrm{e} / \mathrm{h}$ close to 1 and therefore eliminate a constant term (except for calibration errors) in the resolution. Silicon has many advantages for calibration. The proposed detector would be superior to some other approaches in calorimetry and electron and muon measurement, but it is unlikely to be compatible with large magnetic tracking.

We have pointed out the considerable value, for hadron-hadron colliders, of making the calorimeter truly hermetic, and have suggested ways of 'calorimetrizing the machine' to achieve this.

\section{Acknowledgements}

We thank J. Ellis, D. Froidevaux, G. Gilchriese, P. Jenni, B. Montague, F. Richard and W. Willis for helpful discussions. We also thank the organizers, and especially J. Mulvey, for the arrangement of a very pleasant workshop in the Italian Alps. 


\section{REFERENCES}

[1] P. Jenni et al. (LHC Jet Study Group), Proc. ECFA-CERN Workshop on a Large Hadron Collider in the LEP Tunnel, Lausanne and CERN, 1984 (ECFA 84/85, CERN 84-10, Geneva, 1984), p. 165.

[2] Proc. 1984 Summer Study on the Design and Utilization of the Superconducting Supercollider, Snowmass, Colo., 1984, eds. R. Donaldson and J. Morfin (AIP, New York, 1985).

Proc. 1986 Summer Study on the Physics of the Superconducting Supercollider, Snowmass, Colo., 1986, eds.

R. Donaldson and J. Marx, in preparation.

[3] P.N. Burrows and G. Ingelman, Univ. Oxford preprint 1/87 (1987), paper contributed to the Workshop on Physics at Future Accelerators, La Thuile, 1987.

[4] R.K. Böck et al., Nucl. Instrum. Methods 186 (1983) 533.

[5] R. Brun et al., Group report CERN-DD/EE/84-1 (1984).

[6] A. Beer et al., Nucl. Instrum. Methods 224 (1984) 360.

[7] M. Albrow et al., preprint CERN-EP/87-55 (1987).

[8] R. Wigmans, preprint CERN/EF/86-18 (1986).

[9] C.W. Fabjan et al., Nucl. Instrum. Methods 141 (1977) 61.

[10] J. Brau and A. Gabriel, Nucl. Instrum. Methods A238 (1985) 489.

[11] G. Barbiellini et al., Nucl. Instrum. Methods A235 (1985) 55.

G. Barbiellini et al., Nucl. Instrum. Methods A236 (1985) 316.

. A. Nakamato et al., Nucl. Instrum. Methods A238 (1985) 53.

A. Nakamato et al., Nucl. Instrum. Methods A251 (1986) 275.

[12] P.G. Rancoita and A. Seidman, preprint CERN-EP/86-113 (1986).

[13] V. Cherniatin et al., report CERN-HELIOS-171 (1986).

[14] W.R. Nelson, H. Hirayama and D.W.O. Royers, SLAC Report 165 (1985).

[15] G. Lutz et al., to appear in Proc. 1st Int. Conf. on Fast Analog Electronics for High-Energy Physics, Philadelphia, 1987.

H. Spieler et al., ibid.

J. Melean et al., ibid.

[16] J. Walker and S. Parker, private communication, March 1987.

[17] M. Campanella et al., Nucl. Instrum. Methods A243 (1986) 93.

[18] P. Borgeaud et al., Nucl. Instrum. Methods 211 (1983) 363.

[19] P.A. Aarnio, A. Fassó, H.J. Möhring, J. Ranft and G.R. Stevenson, FLUKA86 User's Guide, report CERN TIS-RP/168 (1986).

[20] C. Leroy et al., preprint CERN-EP/86-66 (1986).

[21] H.U. Bengtsson, G. Ingelman and T. Sjöstrand, PYTHIA version 4.1, The Lund Monte Carlo for QCD

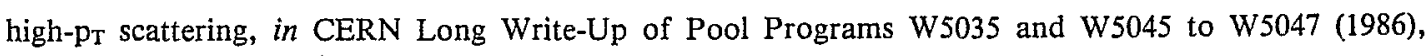
p. 104.

[22] T.A. Gabriel, Oak Ridge Nat. Lab. report ORNL-TM-9727, presented at the CERN Workshop on Shower Simulation for LEP Experiments, Geneva, 1985.

[23] M.B. Emmett, Oak Ridge Nat. Lab. report ORNL-4972 (1975).

[24] S. Battisti, R. Bossart, H. Schönbacher and M. Van de Voorde, CERN 75-18 (1975).

[25] F. Wulf, D. Brauing and W. Gaebler, Data compilation of irradiation tested electronic components, Hahn-Meitner Institute for Nuclear Research (Berlin) report TN 53/08 (1st edition 1981), Vols. 1-3 (2nd editions: 1981, 1983, and 1984, respectively).

[26] H. Beger, report CERN-SPS/ARF/77-21 (1977).

[27] P. Beynel, P. Maier and H. Schönbacher, CERN 82-10 (1982).

[28] T. Sjöstrand and M. Bengtsson, Lund report LU TP 86-22 (1986).

[29] T. Sjöstrand, Comput. Phys. Commun. 28 (1983) 229. 